\title{
A Systematic Review and Meta-analysis on Bolton's ratios: Normal occlusion and Malocclusion
}

\author{
Vanessa Machado ${ }^{1,2 *}$, João Botelho², Paulo Mascarenhas², José João Mendes², Ana Delgado ${ }^{1,2}$
}

${ }^{1}$ Orthodontics Department, Clinical Research Unit (CRU), Centro de Investigação Interdisciplinar Egas Moniz (CiiEM), Egas Moniz - Cooperativa de Ensino Superior, C.R.L., Monte de Caparica, Almada, Portugal

${ }^{2}$ Clinical Research Unit (CRU), Centro de Investigação Interdisciplinar Egas Moniz (CiiEM), Egas Moniz - Cooperativa de Ensino Superior, C.R.L., Monte de Caparica, Almada, Portugal

\author{
*Author responsible for correspondence - Vanessa de Almeida Machado \\ Address: Campus Universitário, Quinta da Granja, Monte de Caparica. \\ Telephone number: + 351964805122 \\ E-mail address: vmachado@egasmoniz.edu.pt
}

\begin{abstract}
Introduction: The purposes of this study were to seek for overall ratio (OR) and anterior ratio (AR) patients data in normal occlusion and Angle's malocclusion studies, and to assess if such results support Bolton's standards as general references.

Methods: Pubmed, Medline, CENTRAL and Scholar databases were searched up to February 2018 (CRD42018088438). Gray literature was explored through OpenGray. Non-randomized clinical studies, published in English and assessing Bolton's OR and AR in normal occlusion and Angle's malocclusion groups (Class I, Class II, Class II division 1, Class 2 division 2, Class III) patients were included. OR and AR means and standard deviations (SD) were collected. Potential covariates (study design, publication year, country where the study was conducted, number of cases, gender, mesiodistal measurement method, and calibration method) were also extracted. The National Health Heart Lung, and Blood Institute's Quality Assessment Tool for Observational Cohort and Cross-Sectional Studies was used to assess each included studies quality. Pairwise Random-Effects and Multilevel Bayesian Network Meta-Analyses were used to synthesize available data.
\end{abstract}

Results: Fifty-two observational studies were included (8872 participants; male/females 2674/3272; 16 studies lacked gender information). For normal occlusion, global pooled estimates for OR and AR means were $91.74 \%$ (95\% Cl: 91.37-92.10) and 78.24\% (95\% Cl: 77.85-78.63), respectively. We could identify on Angle's Class III patients meaningful OR and AR mean deviations from normal occlusion $(0.89,95 \%$ credible interval [Crl], 0.661.12, and $0.66,95 \% \mathrm{Crl}, 0.38-0.94$, respectively), while on Class I patients we found a meaningful mean deviation from normal occlusion only for OR $(0.25,95 \% \mathrm{Crl}, 0.03-0.47)$. Concerning gender impact, male patients presented higher $\mathrm{OR}(0.30,95 \% \mathrm{Cl} 0.00-0.59)$ and $\mathrm{AR}(0.41,95 \% \mathrm{Cl} 0.00-0.83)$ mean values than females in Class I.

Conclusions: The results show that global pooled $O R$ and $A R$ mean values for normal occlusion patients are slightly above Bolton's original values. Class I, for OR mean values, and Class III, for both OR and AR, are proportionally larger than normal occlusion patients. Gender had almost no impact on teeth mesiodistal proportion.

Keywords: Tooth size; Tooth size discrepancy; Bolton ratios; Meta-analysis; Systematic review 


\section{Introduction}

As once mentioned, an appropriate balance of mesiodistal tooth widths between maxillary and mandibular arches allows for a proper interdigitation, overbite, and overjet in a normal occlusion, with the best possible esthetic and function (1). Currently, the extent of mesiodistal movement roots clinical practice from conventional treatment to orthodontic aligners concept and has allured clinical interest, particularly in anteroposterior malocclusions correction $(2,3)$.

The concept of a proportional balance between the mesiodistal sums of maxillary and mandibular teeth may have had its origins in the geometric theories of dental articulation previously proposed. In 1899, Bonwill (4) attempted to develop a geometric theory of occlusion, stating that "Nature left to herself, always brings proposition... the proportions of upper teeth to the lower teeth are as exact as any". This nature theory was pervasive in early orthodontics and was seen in the strict non-extraction period started by Edward Angle. Firmly, the mesiodistal widths of teeth were initially investigated by Black (5). Historically, Young (6) was the first to devote attention to intermaxillary tooth width ratio in occlusion, and, thereafter, Gilpatric (7) found that the upper arch was 8 to 12 millimeters wider than the lower arch. Over the years, to account and aware this proportion, several methods have been proposed to assess interarch tooth size relationship (1,8-11), but Bolton's ratios have become widely applied in orthodontics' research.

In this regards, the overall ratio (OR) is the percentage obtained by summing the widths of the 12 mandibular teeth divided by the sum of the widths of the 12 maxillary teeth. Also, the anterior ratio (AR) is the percentage obtained by summing the widths of the six mandibular anterior teeth divided by the sum of the widths of the six maxillary anterior teeth $(1,11)$. In average, OR were of $91.3 \%( \pm 1.91)$ and AR were of $77.2 \%( \pm 1.65)$, respectively, and these promptly became standard values in the diagnosis and guidance of orthodontic treatments.

Over time, Bolton's analysis have proved to be clinically useful in extreme teeth size discrepancies. However, without neglecting his valuable contribution, its methodology and conclusions should be carefully evaluated. First, these studies had a potential selection bias since the population was not specified, particularly concerning races, ethnicities, and gender. Second, although the author has stated that his ratios were based on 55 cases "where excellent occlusions existed", 44 models were from patients who have undergone orthodontic treatment, and only 11 were untreated (1).

According to literature, teeth size variation is ethnic- and gender-related $(5,12-16)$ pointing out an anthropological significance with genetic underpinnings $(17,18)$. For this reason, the application of Bolton analyses and the proposed standard values for a harmonious dentition might not be valid for other populations. Therefore, this population-based variation has became a subject of interest for many researchers, which led to the attempt to establish normative standards for different racial groups $(12,14,19-21)$.

Another relevant question is the relationship between the tooth size discrepancy for both OR and AR and the various types of Angle's malocclusion. Although several investigators have emphasized the relationship between Bolton ratios discrepancy and malocclusions in multiple populations $(14,22-26)$, there is no consensus about its correlation with the different types of malocclusions classified by Angle.

\section{Objectives}

No study has investigated, in an evidence-based manner, normative values for mesiodistal proportions from systematically researched worldwide data in normal occlusion and Angle's malocclusions. For that reason, the primary aim of this systematic review was to synthesize global estimates for normal occlusion OR and AR mean values, and to compare such values with those proposed by Bolton, to address the following focused question: are current standards globally appropriate? Secondly, we intended to obtain OR and AR global pooled estimates for each type of Angle's malocclusion and compare them against the obtained values for normal occlusion under a multilevel bayesian network meta-analysis model. 


\section{Materials and Methods}

\section{Protocol and registration}

The protocol for this systematic review was made a priori, agreed upon by all authors and registered in PROSPERO (ID Number: CRD42018088438). This systematic review was conducted according to the Cochrane Handbook (27) and reported according to the PRISMA statement (Preferred Reporting Items for Systematic Reviews and Meta-Analyses) (28) (Supplement Table S1) and its extension for abstracts (29).

There were involved two researchers from the Orthodontics Department, Clinical Research Unit, CiiEM, [Instituto Universitário Egas Moniz]: VM and AD; and three researchers from the Clinical Research Unit, CiiEM, [Instituto Universitário Egas Moniz]: JB, PM and JJM.

\section{Eligibility criteria}

Studies were eligible for inclusion based on the following criteria:

1. Observational studies (randomized and non-randomized study cohort/longitudinal study, crosssectional study).

2. English language studies.

3. human study population.

4. determined Bolton's analysis with normal occlusion, and/or Angle's Class I, Class II, Class II division 1, Class II division 2 and/or Class III, in patients without previous orthodontic treatment.

5. dental casts or digital models with all permanent teeth from the maxillary and mandibular right first molar to the left first molar completely erupted, without tooth deformities, mesiodistal restorations, caries or abrasion that could affect the teeth's mesiodistal diameter.

6. The study measured the largest mesiodistal teeth dimension to the nearest $0.01 \mathrm{~mm}$, through digital caliper or software.

Narrative reviews, case reports, and case series studies were excluded from review.

\section{Search strategy}

A systematic search was conducted and updated in February 2018, covering the following electronic databases: Pubmed, Medline, CENTRAL and Scholar. The strategy used for the electronic search was the following: ["Bolton ratio"OR"tooth size discrepancy"OR"Bolton discrepancy"OR"tooth-size ratios"OR"toothsize measurement"OR"Bolton analysis"].

No limitations were applied regarding publication year. The reference lists of included articles and relevant reviews were manually searched. Gray literature was searched using the latter strategy in OpenGray. Authors were contacted when necessary for additional data or clarifications.

\section{Assessment of Validity}

The eligibility of each study was assessed independently by two investigators (VM and JB), who screened the titles and/or abstracts of retrieved studies. Inclusion was dependent on the following eligibility criteria: randomized or non-randomized trials with OR and/or AR data. Final selection of studies was performed by three authors independently (JB, VM, PM), and verified by a fourth and fifth authors (JJM, AD), by reviewing the full text based on inclusion criteria above. Discussion resolved any disagreements. Non-full papers, such as conference abstracts and letters to editors, were excluded.

\section{Data extraction}

Data were extracted to a predefined table. We used information about: the first author's name, study design, publication year, country and continent where the study was conducted, number of cases and participants, gender, tooth width measurement method, OR and AR (mean and standard deviation). Type of occlusion was classified into normal occlusion, Angle's Class I, Class II (division 1 and division 2) or Class III. Populations were categorized into continental groups: African, American, Asia (including Japanese populations based in Hawaii), European, and Oceania. We extracted Bolton OR, AR means and standard deviations, for both gender, in all selected studies population samples. Concerning additional data/clarifications, we tried to contact corresponding authors (on 23rd of February 2018). 


\section{Quality assessment and Risk of Bias in Included Studies}

Quality Assessment Tool for Observational Cohort and Cross-Sectional Studies statement proposed by National Heart, Lung, and Blood Institute (NIHLBI) was used to appraise study quality (from https://www.nhlbi.nih.gov/health-topics/study-quality-assessment-tools). The checklist was adapted since criteria 7, 8, 10 and 13 did not apply. The reviewers (VM, JB) determined a global quality score for each article. Each methodologic quality criterion was assigned a point, to a total maximum of 10 achievable points. Studies reaching 9 or 10 points were arbitrarily considered of high quality, studies with 7 or 8 points were classified as medium quality, and studies with 6 points or less considered of low methodologic quality. To be included, articles had to be at least of medium quality. Furthermore, data extracted from selected studies were screened for precision inconsistencies, to prevent an unbalanced contribution of some studies data in the meta-analysis.

\section{Summary Measures \& Synthesis of Results}

The objective of synthesis of the normal occlusion OR and AR mean values was accomplished by pairwise random effects meta-analysis using OpenMetaAnalyst (2016) ${ }^{26}$ software. Quantity $I^{2}$ was measured to account for the degree of dispersion of Bolton ratios mean estimates, and the overall homogeneity statistical significance was calculated through the $\chi 2$ test ${ }^{22}$. Funnel plots were used to visualize and quantify metaanalysis publication bias, respectively, if appropriate ${ }^{28-33}$ All tests were two-tailed with alpha set at 0.05 except for homogeneity test whose significance level cutoff was considered to be 0.10 due to the low power of the $\chi 2$ test with a limited amount of studies. Unpaired z-test was used to compare our normal occlusion mean results with Bolton original values, with significance level set at $5 \%$.

In a number of articles ${ }^{22,38-46}$, Class II division 1 and division 2 summary statistics were published separately, and it was necessary to calculate the combined mean $\left(\bar{x}_{12}\right)$ and standard deviation $\left(\sigma_{12}\right)$ for the overall Class II through the following formulas:

$$
\begin{aligned}
& \bar{x}_{12}=\frac{N_{1} \cdot \bar{x}_{1}+N_{2} \cdot \bar{x}_{2}}{N_{1}+N_{2}} \\
& \sigma_{12}=\sqrt{\frac{\left(N_{1}-1\right) \cdot \sigma_{1}^{2}+\left(N_{2}-1\right) \cdot \sigma_{2}^{2}+\frac{N_{1} \cdot N_{2}}{N_{1}+N_{2}} \cdot\left(\bar{x}_{1}^{2}+\bar{x}_{2}^{2}-2 \bar{x}_{1} \bar{x}_{2}\right)}{N_{1}+N_{2}-1}}
\end{aligned}
$$

Similarly, in McSwiney ${ }^{41}$ and Nie ${ }^{47}$ studies, it was published data for surgical and non-surgical in Class III, and we used the aforementioned formula to combine the mean and standard deviation.

To fulfill the second objective, we were required to estimate OR and AR global mean of Angle's malocclusion types (Class I, Class II, Class II division 1, Class II division 2 and Class III) and compare each them against the normal occlusion global mean value. Due to the complexity of the distribution of the extracted data across the selected reports, we aimed to address such issue globally under a multilevel Bayesian Network Meta-Analysis (Bayesian NMA) model. Therefore, we went through Rstan, a R package that inter-connects $\mathrm{R}$ and STAN languages making easy the design and handling of such complex model. Thus, we fitted a model with continuous outcome data, given as the randomly distributed differences between the occlusion classes means (including the normal occlusion class), with normally distributed likelihood and identity as link function. We sourced the initial mean and variance for AR and OR normal occlusion values from the Pairwise Meta-Analysis (Pairwise MA). Furthermore, for every study reporting mean values for several different OR and AR occlusion classes, it was necessary to account for the correlation between the classes differences, when the same class is used, within the same study, as baseline for difference of means determination. Therefore, we modeled all of the classes differences (across all of the studies) using a single multivariate normal distribution with a vector as mean and with a covariance matrix. Some of the covariances were zero, since classes differences sourced from different studies are not correlated. On the other hand,t the covariances for the differences sharing the same reference class within the same study were set to the variance of the shared reference class (for instance the normal occlusion class), as this was the within study "shared" variance. Sampling from the posterior 
distribution was performed by running three Hamiltonian Monte Carlo chains of 100,000 iterations each, after a warmup of 40,000 . The function returned fit statistics that included adjusted estimates and associated credibility intervals ( $\mathrm{Crl} ; 2.5$ to 97.5 percentiles) for all mean values of malocclusion classes and malocclusion classes mean differences to normal occlusion. We performed both random effects and fixed effects bayesian approaches and lately selected the fixed-effects variant results because was the one with the lowest Deviance Information Criteria (DIC).

\section{RESULTS}

\section{Study Selection}

The initial electronic database search resulted in a total of 2700 articles, leaving 2533 articles after the removal of duplicates. No additional relevant articles were identified following a hand search of reference lists. Following title and abstract screening, 178 studies were selected for full-text evaluation. After full-text eligibility assessment, 119 studies were excluded (Supplement Table S2). Five studies were excluded for presenting low quality and high risk of bias (in the Risk of bias across studies section). Two articles only reported data for Class I malocclusion, preventing its inclusion in the Bayesian Network Meta-Analysis. At last, fifty-two studies were included in this review (Figure 1).

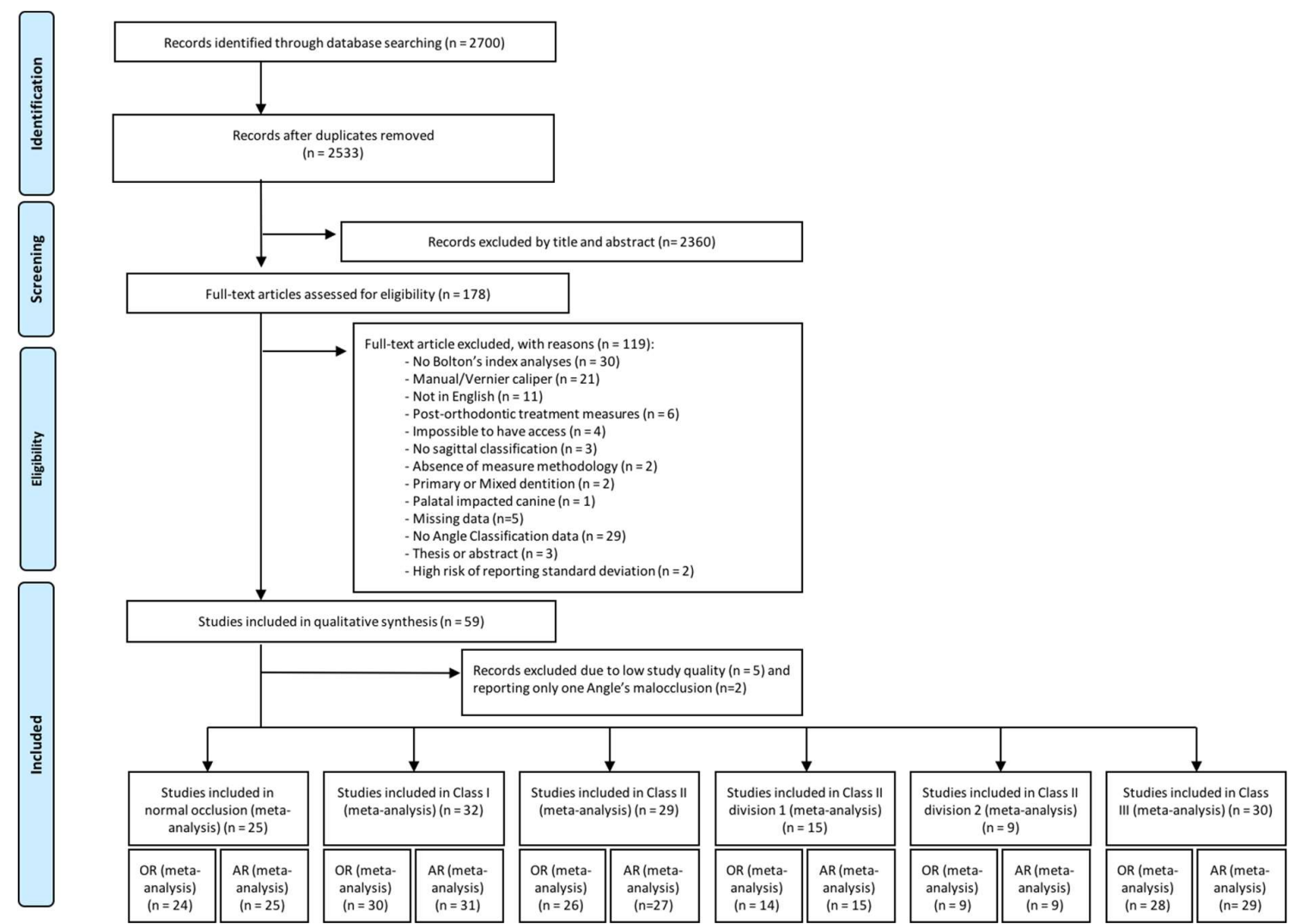

Figure 1: Flowchart of the study selection process.

\section{Study Characteristics Setting}

Table 1 summarizes the characteristics of the included studies. Globally, the analysis included 8872 participants (2614 men and 3272 women). However, sixteen studies $(25,26,30-43)$ lacked gender information (2986 participants). Additionally, two multicentre study $(12,34)$ included samples from 2 and 3 different 
countries and from different continents and, consequently, they were counted as three samples, although in Lavelle (12) the author did not specify the African country preventing it from being analyzed in metaregression.

Table 1 and Figure 2 shows that only one study with normal occlusion subjects (12) was published in the 70', and thereafter was a lack of published reports for almost 30 years. Additionally, the first author that investigated the Bolton ratios in Angle's malocclusion groups was Crosby at 1989 (39), more than 30 years after Bolton's article (1). After 1999 larger datasets were published on different continents. Fifty-two crosssectional studies from four different continents, namely Africa $(12,30,34,36,44-46)$, Asia $(12,24,25,31,32,34,35,37,38,40-42,47-58)$, South America $(26,33,39,59-66)$, and Europe $(12,22,43,67-74)$ were included in the qualitative synthesis. Lavelle's (12) and Al-Duliamy's (34) were multicentric studies that comprised European, Asian, and African subjects, and Asian and African participants, respectively. Notably, no study was performed in Oceania nor North America. Due to the inadequacy of continent representation, continent subgroup analysis was not conceivable to perform. 
Table 1: Baseline characteristics for studies included in pairwise meta-analysis and Bayesian network meta-analysis.

\begin{tabular}{|c|c|c|c|c|c|c|c|c|c|c|}
\hline $\begin{array}{c}\text { Study (Year) } \\
\text { (Country) (City) }\end{array}$ & Continent & Subjets (N) & $\begin{array}{c}\text { Female / } \\
\text { Male }\end{array}$ & Method & Total OR (SD) & $\begin{array}{c}\text { Total } \\
\text { AR (SD) }\end{array}$ & $\begin{array}{c}\text { Female / Male } \\
\text { OR (SD) }\end{array}$ & $\begin{array}{c}\text { Female / Male } \\
\text { AR (SD) }\end{array}$ & $\begin{array}{l}\text { Included in } \\
\text { Pairwise } \\
\text { Meta-analysis }\end{array}$ & $\begin{array}{c}\text { Included in } \\
\text { Bayesian } \\
\text { network meta- } \\
\text { analysis }\end{array}$ \\
\hline & & & & Normal occlusion & & & & & & \\
\hline $\begin{array}{l}\text { Machado et al. } \\
\text { (2018) (Portugal) } \\
\text { (Almada) }\end{array}$ & Europe & 29 & $10 / 19$ & $\begin{array}{l}\text { Digital caliper }(0,01 \\
\mathrm{mm})\end{array}$ & $92.1(2.2)$ & $\begin{array}{l}78.4 \\
(3.5)\end{array}$ & $\begin{array}{l}91.7(2.2) / \\
92.9(2.1)\end{array}$ & $\begin{array}{c}77.9(3.1) / 79.3 \\
(4.1)\end{array}$ & Yes & Yes \\
\hline $\begin{array}{l}\text { Patel et al. (2017) } \\
\text { (India) (Pune) }\end{array}$ & Asia & 50 & $25 / 25$ & $\begin{array}{l}\text { Digital caliper }(0,01 \\
\mathrm{mm})\end{array}$ & $92.73(2.69)$ & $\begin{array}{c}80.8 \\
(2.86)\end{array}$ & $\begin{array}{l}91.92(3.4) / \\
93.55(1.36)\end{array}$ & $\begin{array}{c}80.34(3.27) / \\
80.6(2.45)\end{array}$ & Yes & No \\
\hline $\begin{array}{c}\text { Sakoda et al. } \\
\text { (2016) (Brazil) (São } \\
\text { Paulo) }\end{array}$ & America & 90 & $45 / 45$ & $\begin{array}{l}\text { Digital caliper }(0,01 \\
\mathrm{mm})\end{array}$ & $91.63(1.95)$ & $\begin{array}{l}77.57 \\
(2.45)\end{array}$ & $\begin{array}{l}91.35(1.87) / \\
91.91(2.04)\end{array}$ & $\begin{array}{l}77.3(2.28) / \\
77.85(2.64)\end{array}$ & Yes & No \\
\hline $\begin{array}{c}\text { Shahid et al. (2016) } \\
\text { (Pakistan) (different } \\
\text { states) }\end{array}$ & Asia & 128 & $64 / 64$ & $\begin{array}{l}\text { Digital caliper }(0,01 \\
\mathrm{mm})\end{array}$ & $92.8(2.79)$ & $\begin{array}{l}79.25 \\
(3.81)\end{array}$ & $\begin{array}{l}93.1(2.4) / \\
92.5(3.1)\end{array}$ & $\begin{array}{c}79.5(3.6) / 79 \\
(4.4)\end{array}$ & Yes & No \\
\hline $\begin{array}{c}\text { Lombardo et al. } \\
\text { (2016) (USA) (New } \\
\text { York) }\end{array}$ & Europe & 56 & $22 / 34$ & $\begin{array}{l}\text { Intraoral Scanner } \\
\text { (3shape) }\end{array}$ & $91.56(2)$ & $\begin{array}{l}77.65 \\
(2.46)\end{array}$ & $\begin{array}{l}91.55(2) / \\
91.57(2)\end{array}$ & $\begin{array}{c}77.88(2) / 77.3 \\
\text { (3) }\end{array}$ & Yes & No \\
\hline $\begin{array}{l}\text { Chugh et al. (2015) } \\
\text { (India) (Lucknow) }\end{array}$ & Asia & 50 & $25 / 25$ & $\begin{array}{l}\text { Digital caliper }(0,01 \\
\mathrm{mm})\end{array}$ & $91.88(1.99)$ & $\begin{array}{l}79.64 \\
(2.61)\end{array}$ & $\begin{array}{l}91.8(2.34) / \\
91.96(1.63)\end{array}$ & $\begin{array}{l}79.16(2.23) / \\
80.12(1.73)\end{array}$ & Yes & Yes \\
\hline $\begin{array}{l}\text { Bugaighis et al. } \\
\text { (2015) (Libya) } \\
\text { (Benghazi) }\end{array}$ & Africa & 15 & NS & $\begin{array}{c}\text { Digital caliper }(0,01 \\
\mathrm{mm})\end{array}$ & $90.24(1.89)$ & $\begin{array}{l}76.88 \\
(2.42)\end{array}$ & NS & NS & Yes & Yes \\
\hline $\begin{array}{l}\text { Ismail et al. (2015) } \\
\text { (Sudan) } \\
\text { (Khartoum) }\end{array}$ & Africa & 55 & $25 / 30$ & Digital caliper $(0,1 \mathrm{~mm})$ & $91.47(2.83)$ & $\begin{array}{l}77.46 \\
(3.16)\end{array}$ & $\begin{array}{c}91.25(2.94) / \\
91.73(2.9)\end{array}$ & $\begin{array}{l}77.22(3.43) / \\
77.73(2.82)\end{array}$ & Yes & Yes \\
\hline $\begin{array}{l}\text { Hashim et al. } \\
(2015) \text { (Sudan) } \\
\text { (Khartoum) }\end{array}$ & Africa & 60 & $30 / 30$ & $\begin{array}{c}\text { Digital caliper }(0,01 \\
\mathrm{mm})\end{array}$ & $90.8(3.5)$ & $\begin{array}{l}76.9 \\
(3.6)\end{array}$ & $\begin{array}{c}90.6(3.1) / 91 \\
(3.9)\end{array}$ & $\begin{array}{c}77(3.7) / 76.9 \\
(3.6)\end{array}$ & Yes & No \\
\hline $\begin{array}{l}\text { Ricci et al. (2013) } \\
\text { (Brazil) (São Paulo) }\end{array}$ & America & 35 & NS & $\begin{array}{l}\text { Digital caliper }(0,01 \\
\mathrm{mm})\end{array}$ & $90.38(1.58)$ & $\begin{array}{c}77.49 \\
(2.2)\end{array}$ & $\begin{array}{l}90.36(1.7) / \\
90.44(1.2)\end{array}$ & $\begin{array}{l}77.73(2.39) / \\
76.68(1.19)\end{array}$ & Yes & Yes \\
\hline $\begin{array}{l}\text { Celikoglu et al. } \\
\text { (2013) (Turkey) } \\
\text { (Karadeniz Ereğli) }\end{array}$ & Europe & 26 & $14 / 12$ & СBCT & $90.69(2.21)$ & $\begin{array}{l}77.58 \\
(2.71)\end{array}$ & NS & NS & Yes & No \\
\hline $\begin{array}{l}\text { Jóias et al. (2011) } \\
\text { (Brazil) (São Paulo) }\end{array}$ & America & 35 & $8 / 27$ & $\begin{array}{l}\text { Digital caliper }(0,01 \\
\mathrm{mm})\end{array}$ & NS & $\begin{array}{l}77.48 \\
(2.22)\end{array}$ & NS & $\begin{array}{c}77.61(2.45) / \\
77.05(1.1)\end{array}$ & Yes & No \\
\hline $\begin{array}{l}\text { Fernandes et al. } \\
\text { (2011) (Brazil) } \\
\text { (Bauru) }\end{array}$ & America & 140 & $70 / 70$ & $\begin{array}{l}\text { Digital caliper }(0,01 \\
\mathrm{mm})\end{array}$ & $91.32(1.98)$ & $\begin{array}{c}77 \\
(2.71)\end{array}$ & $\begin{array}{c}90.87(1.94) / \\
91.77(1.96)\end{array}$ & $\begin{array}{l}76.54(2.79) / \\
77.46(2.61)\end{array}$ & Yes & No \\
\hline $\begin{array}{l}\text { Manopatanakul et } \\
\text { al. (2011) } \\
\text { (Thailand) } \\
\text { (Bangkok) }\end{array}$ & Asia & 37 & NS & $\begin{array}{l}\text { Digital caliper }(0,01 \\
\mathrm{mm})\end{array}$ & $91.66(1.74)$ & $\begin{array}{l}77.09 \\
(2.18)\end{array}$ & NS & NS & Yes & No \\
\hline $\begin{array}{l}\text { Lee et al. (2011) } \\
\text { (South Korea) }\end{array}$ & Asia & 307 & $\begin{array}{c}188 / \\
119\end{array}$ & $\begin{array}{c}\text { Digital caliper }(0,01 \\
\mathrm{mm})\end{array}$ & $90.42(1.94)$ & $\begin{array}{l}77.54 \\
(2.54)\end{array}$ & $\begin{array}{c}90.3(2) / 90.5 \\
(1.9)\end{array}$ & $\begin{array}{c}77.6(2.6) / 77.5 \\
(2.5)\end{array}$ & Yes & No \\
\hline
\end{tabular}


Peer-reviewed version available at Journal of Orthodontics 2019; doi:10.1177/1465312519886322

(Seoul)

\begin{tabular}{|c|c|c|c|c|c|c|c|c|c|c|}
\hline $\begin{array}{l}\text { Oktay et al. (2010) } \\
\text { (Turkey) (Erzurum) }\end{array}$ & Europe & 100 & $61 / 39$ & RMI 550 3D (0,01mm) & $92.1(1.95)$ & $\begin{array}{l}79.28 \\
(2.53)\end{array}$ & $\begin{array}{l}91.63(2.04) / \\
92.39(1.84)\end{array}$ & $\begin{array}{l}79.17(2.65) / \\
79.35(2.47)\end{array}$ & Yes & Yes \\
\hline $\begin{array}{l}\text { Jóias et al. (2010) } \\
\text { (Brazil) (São Paulo) }\end{array}$ & America & 35 & $8 / 27$ & $\begin{array}{l}\text { Intraoral Scanner } \\
\text { (3shape) }\end{array}$ & $91.58(2.2)$ & $\begin{array}{l}78.66 \\
(2.72)\end{array}$ & NS & NS & Yes & No \\
\hline $\begin{array}{l}\text { Freire et al. (2007) } \\
\text { (Brazil) (Rio de } \\
\text { Janeiro) }\end{array}$ & America & 30 & $15 / 15$ & $\begin{array}{l}\text { Digital caliper }(0,01 \\
\mathrm{mm})\end{array}$ & $91.46(1.63)$ & $\begin{array}{l}77.83 \\
(2.19)\end{array}$ & NS & NS & Yes & No \\
\hline $\begin{array}{l}\text { Endo et al. (2007) } \\
\text { (Japan) (Niigata) }\end{array}$ & Asia & 60 & $30 / 30$ & $\begin{array}{l}\text { Digital caliper }(0,01 \\
\mathrm{mm})\end{array}$ & $91.6(2.11)$ & $\begin{array}{l}78.39 \\
(2.18)\end{array}$ & $\begin{array}{l}91.69(2.35) / \\
91.51(1.88)\end{array}$ & $\begin{array}{l}78.57(2.19) / \\
78.21(2.18)\end{array}$ & Yes & No \\
\hline $\begin{array}{c}\text { Ciger et al. (2006) } \\
\text { (Turkey) } \\
\text { (Hacettepe) }\end{array}$ & Europe & 125 & $55 / 70$ & $\begin{array}{l}\text { Digital caliper }(0,01 \\
\mathrm{mm})\end{array}$ & $91.95(2.2)$ & $\begin{array}{l}77.95 \\
(2.35)\end{array}$ & $\begin{array}{l}91.82(1.99) / \\
91.97(1.65)\end{array}$ & $\begin{array}{l}78.43(2.41) / \\
78.62(2.24)\end{array}$ & Yes & Yes \\
\hline $\begin{array}{l}\text { Carreiro et al. } \\
\text { (2005) (Brazil) } \\
\text { (Paraná) }\end{array}$ & America & 41 & $20 / 21$ & Microscribe 3DX & $91.76(2.51)$ & $\begin{array}{c}78.24 \\
(3.4)\end{array}$ & NS & NS & Yes & Yes \\
\hline $\begin{array}{l}\text { Uysal et al. (2005) } \\
\text { (Turkey) (Konya) }\end{array}$ & Europe & 150 & $72 / 78$ & $\begin{array}{l}\text { Digital caliper }(0,01 \\
\mathrm{mm})\end{array}$ & $91.9(3.21)$ & $\begin{array}{l}78.56 \\
(3.23)\end{array}$ & $\begin{array}{l}91.73(2.26) / \\
89.83(2.33)\end{array}$ & $\begin{array}{l}78.33(2.42) / \\
78.18(2.82)\end{array}$ & Yes & Yes \\
\hline $\begin{array}{c}\text { Alkofide et al. } \\
\text { (2002) (Saudi } \\
\text { Arabia) (Jeddah) }\end{array}$ & Asia & 60 & NS & $\begin{array}{l}\text { Digital caliper }(0,01 \\
\mathrm{mm})\end{array}$ & $93.58(2.12)$ & $\begin{array}{l}78.86 \\
(2.55)\end{array}$ & $\begin{array}{l}92.36(2.37) / \\
92.12(1.67)\end{array}$ & $\begin{array}{l}78.79(3.19) / \\
78.75(2.27)\end{array}$ & Yes & Yes \\
\hline $\begin{array}{l}\text { Nie et al. (1999) } \\
\text { (China) (Beijing) }\end{array}$ & Asia & 60 & $30 / 30$ & Software & $93.27(2.48)$ & $\begin{array}{l}81.52 \\
(2.82)\end{array}$ & $\begin{array}{l}93.11(2.64) / \\
93.44(2.35)\end{array}$ & $\begin{array}{l}81.1(2.27) / \\
81.95(2.28)\end{array}$ & Yes & Yes \\
\hline & Europe & 40 & $20 / 20$ & Digital caliper $(0,1 \mathrm{~mm})$ & $91.25(2)$ & $\begin{array}{l}77.15 \\
(1.6)\end{array}$ & $\begin{array}{c}90.8(1.85) / \\
91.7(2.04)\end{array}$ & $\begin{array}{l}77.5(1.62) / \\
76.8(1.49)\end{array}$ & Yes & No \\
\hline Lavelle et al. (1972) & Africa & 40 & $20 / 20$ & Digital caliper $(0,1 \mathrm{~mm})$ & $93.2(2.11)$ & $\begin{array}{c}79 \\
(2.02)\end{array}$ & $\begin{array}{c}92.9(1.78) / \\
93.5(2.35)\end{array}$ & $\begin{array}{l}78.6(1.89) / / \\
79.4(2.06)\end{array}$ & Yes & No \\
\hline & Asia & 40 & $20 / 20$ & Digital caliper $(0,1 \mathrm{~mm})$ & $92.75(1.53)$ & $\begin{array}{l}78.45 \\
(1.55)\end{array}$ & $\begin{array}{c}92.1(1.55) / \\
92.6(2.47)\end{array}$ & $\begin{array}{l}78.2(1.38) / \\
78.7(1.66)\end{array}$ & Yes & No \\
\hline & & & & Class I & & & & & & \\
\hline $\begin{array}{l}\text { Machado et al. (2018) } \\
\text { (Portugal) (Almada) }\end{array}$ & Europe & 50 & $29 / 21$ & Digital caliper $(0,01 \mathrm{~mm})$ & $92.90(2.70)$ & $\begin{array}{l}79.30 \\
(4.00)\end{array}$ & $\begin{array}{l}93.4(2.30) / \\
92.50(2.90)\end{array}$ & $\begin{array}{l}79.60(2.90) / \\
79.00(4.60)\end{array}$ & - & Yes \\
\hline $\begin{array}{l}\text { Saritha et al. (2017) } \\
\text { (India) (Telangana) }\end{array}$ & Asia & 168 & $110 / 58$ & Digital caliper $(0,01 \mathrm{~mm})$ & $92.38(1.86)$ & $\begin{array}{l}79.37 \\
(2.98)\end{array}$ & $\begin{array}{c}92.39(1.95) / \\
92.38(1.82)\end{array}$ & $\begin{array}{l}79.49(2.37) / \\
79.30(3.27)\end{array}$ & - & Yes \\
\hline $\begin{array}{l}\text { Mahmoud et al. } \\
\text { (2017) (Sudan) } \\
\text { (Khartoum) }\end{array}$ & Asia & 52 & NS & Digital caliper $(0,05 \mathrm{~mm})$ & $91.37(2.98)$ & $\begin{array}{l}78.44 \\
(2.91)\end{array}$ & NS & NS & - & Yes \\
\hline $\begin{array}{l}\text { Elsheikhi et al. } \\
\text { (2017) (Libya) } \\
\text { (Benghazi) }\end{array}$ & Africa & 20 & $10 / 10$ & Digital caliper $(0,01 \mathrm{~mm})$ & $89.91(1.79)$ & $\begin{array}{l}74.42 \\
(2.06)\end{array}$ & NS & NS & - & Yes \\
\hline $\begin{array}{l}\text { Cançado et al. (2016) } \\
\text { (Brazil) (Dourados) }\end{array}$ & America & 321 & NS & Digital caliper $(0,01 \mathrm{~mm})$ & $91.61(2.04)$ & $\begin{array}{l}78.37 \\
(2.68)\end{array}$ & NS & NS & - & Yes \\
\hline $\begin{array}{l}\text { Al-Duliamy Iraq et } \\
\text { al. (2016) (Iraq) } \\
\text { (Baghdad) }\end{array}$ & Asia & 70 & NS & Digital caliper $(0,01 \mathrm{~mm})$ & $91.23(2.20)$ & $\begin{array}{l}78.72 \\
(4.53)\end{array}$ & NS & NS & - & Yes \\
\hline $\begin{array}{l}\text { Al-Duliamy Egypt et } \\
\text { al. (2016) (Egypt) }\end{array}$ & Africa & 70 & NS & Digital caliper $(0,01 \mathrm{~mm})$ & $91.63(2.58)$ & $\begin{array}{l}78.85 \\
(2.79)\end{array}$ & NS & NS & - & Yes \\
\hline
\end{tabular}


(Cairo)

\begin{tabular}{|c|c|c|c|c|c|c|c|c|c|c|}
\hline $\begin{array}{l}\text { Chugh et al. (2015) } \\
\text { (India) (Lucknow) }\end{array}$ & Asia & 50 & $25 / 25$ & Digital caliper $(0,01 \mathrm{~mm})$ & $93.06(2.28)$ & $\begin{array}{l}79.6 \\
(3.02)\end{array}$ & $\begin{array}{l}93.35(2.31) / \\
92.79(2.28)\end{array}$ & $\begin{array}{l}80.17(3.13) / \\
79.09(2.92)\end{array}$ & - & Yes \\
\hline $\begin{array}{l}\text { Shastri et al. (2015) } \\
\text { (India (North)) } \\
\text { (Lucknow) }\end{array}$ & Asia & 40 & NS & Digital caliper & $91.73(3.6)$ & $\begin{array}{l}76.89 \\
(4.16)\end{array}$ & NS & NS & - & Yes \\
\hline $\begin{array}{l}\text { Bughaighis et al. } \\
\text { (2015) (Libya) } \\
\text { (Benghazi) }\end{array}$ & Africa & 220 & NS & Digital caliper $(0,01 \mathrm{~mm})$ & $91.55(2.4)$ & $\begin{array}{l}78.29 \\
(2.53)\end{array}$ & NS & NS & - & Yes \\
\hline $\begin{array}{l}\text { Ismail et al. (2015) } \\
\text { (Sudan) (Khartoum) }\end{array}$ & Africa & 49 & $26 / 23$ & Digital caliper $(0,1 \mathrm{~mm})$ & $91.47(2.83)$ & $\begin{array}{l}77.46 \\
(3.16)\end{array}$ & $\begin{array}{l}91.51(3.27) / \\
91.39(2.54)\end{array}$ & $\begin{array}{l}77.00(4.65) / \\
76.55(3.34)\end{array}$ & - & Yes \\
\hline $\begin{array}{l}\text { Maurya et al. (2015) } \\
\text { (India) (Madhya } \\
\text { Pradesh) }\end{array}$ & Asia & 60 & $30 / 30$ & Digital caliper $(0,01 \mathrm{~mm})$ & $92.38(2.51)$ & $\begin{array}{l}80.13 \\
(3.48)\end{array}$ & $\begin{array}{l}93.03(2.34) / \\
91.72(2.58)\end{array}$ & $\begin{array}{c}80.86(3.28) / \\
79.40(3.64)\end{array}$ & - & Yes \\
\hline $\begin{array}{l}\text { Zerouaoui et al. } \\
\text { (2014) (Morocco) } \\
\text { (Rabat) }\end{array}$ & Africa & 30 & NS & Digital caliper & $91.37(2.05)$ & $\begin{array}{l}77.93 \\
(2.60)\end{array}$ & NS & NS & - & Yes \\
\hline $\begin{array}{l}\text { Jindal et al. (2013) } \\
\text { (India) (Punjab) }\end{array}$ & Asia & 300 & $\begin{array}{c}150 / \\
150\end{array}$ & Digital caliper $(0,01 \mathrm{~mm})$ & $92.75(3.15)$ & $\begin{array}{l}79.82 \\
(3.85)\end{array}$ & $\begin{array}{l}93.93(3.34) / \\
91.58(2.44)\end{array}$ & $\begin{array}{c}80.87(43135) / \\
78.77(3.38)\end{array}$ & - & Yes \\
\hline $\begin{array}{l}\text { Asma et al. (2013) } \\
\text { (Malaysia) (Selangor) }\end{array}$ & Asia & 50 & NS & Digital caliper $(0,01 \mathrm{~mm})$ & NS & $\begin{array}{l}78.83 \\
(4.06)\end{array}$ & NS & NS & - & Yes \\
\hline $\begin{array}{l}\text { Ricci et al. (2013) } \\
\text { (Brazil) (São Paulo) }\end{array}$ & America & 35 & NS & Digital caliper $(0,01 \mathrm{~mm})$ & $91.19(2.70)$ & $\begin{array}{l}78.16 \\
(2.87)\end{array}$ & $\begin{array}{l}91.25(3.24) / \\
91.17(2.58)\end{array}$ & $\begin{array}{l}78.66(3.64) / \\
78.01(2.66)\end{array}$ & - & Yes \\
\hline $\begin{array}{l}\text { Ali Hyder et al. } \\
\text { (2012) (Bangladesh) } \\
\text { (Dhaka) }\end{array}$ & Asia & 40 & $20 / 20$ & Digital caliper $(0,01 \mathrm{~mm})$ & $90.40(2.69)$ & $\begin{array}{l}77.70 \\
(2.81)\end{array}$ & $\begin{array}{l}89.82(3.06) / \\
91.06(2.18)\end{array}$ & $\begin{array}{c}77.92(2.80) / \\
77.49(2.87)\end{array}$ & - & Yes \\
\hline $\begin{array}{l}\text { Kansal et al. (2012) } \\
\text { (India) (Karnataka) }\end{array}$ & Asia & 231 & NS & Digital caliper $(0,01 \mathrm{~mm})$ & $91.80(3.30)$ & $\begin{array}{l}79.20 \\
(3.80)\end{array}$ & NS & NS & - & Yes \\
\hline $\begin{array}{l}\text { O'Mahony et al. } \\
\text { (2011) (Ireland) } \\
\text { (Cork) }\end{array}$ & Europe & 60 & $30 / 30$ & OrhoAnalyzer (Software) & $92.30(2.20)$ & $\begin{array}{c}79.00 \\
(43376)\end{array}$ & $\begin{array}{l}92.4(2.20) / \\
92.10(2.20)\end{array}$ & $\begin{array}{l}78.40(2.90) / \\
79.60(3.20)\end{array}$ & - & Yes \\
\hline $\begin{array}{l}\text { Vela et al. (2011) } \\
\text { (USA) (Texas) }\end{array}$ & America & 207 & $110 / 97$ & Digital caliper $(0,01 \mathrm{~mm})$ & NS & $\begin{array}{l}78.97 \\
(2.29)\end{array}$ & NS & $\begin{array}{c}79.12(1.99) / \\
78.84(2.49)\end{array}$ & - & No \\
\hline $\begin{array}{l}\text { Endo et al. (2010) } \\
\text { (Japan) (Niigata) }\end{array}$ & Asia & 66 & $33 / 33$ & Digital caliper $(0,01 \mathrm{~mm})$ & $91.10(2.20)$ & NS & $\begin{array}{l}91.18(2.27) / \\
91.01(2.17)\end{array}$ & NS & - & Yes \\
\hline $\begin{array}{l}\text { Oktay et al. (2010) } \\
\text { (Turkey) (Erzurum) }\end{array}$ & Europe & 100 & $65 / 35$ & RMI 550 3D (0,01 mm) & $92.27(2.16)$ & $\begin{array}{l}78.61 \\
(2.80)\end{array}$ & $\begin{array}{l}92.33(1.88) / \\
92.24(2.32)\end{array}$ & $\begin{array}{l}78.66(2.41) / \\
78.58(3.01)\end{array}$ & - & Yes \\
\hline $\begin{array}{l}\text { Strujić et al. (2009) } \\
\text { (Croatia) (Zagreb) }\end{array}$ & Europe & 110 & $68 / 42$ & Digital caliper $(0,01 \mathrm{~mm})$ & $91.81(1.99)$ & $\begin{array}{l}78.25 \\
(2.58)\end{array}$ & NS & NS & - & Yes \\
\hline $\begin{array}{l}\text { Endo et al. (2009) } \\
\text { (Japan) (Niigata) }\end{array}$ & Asia & 101 & $59 / 42$ & Digital caliper $(0,01 \mathrm{~mm})$ & $91.15(2.14)$ & $\begin{array}{l}77.84 \\
(2.46)\end{array}$ & $\begin{array}{l}91.14(2.33) / \\
91.15(1.99)\end{array}$ & $\begin{array}{l}77.97(2.55) / \\
77.74(2.39)\end{array}$ & - & Yes \\
\hline $\begin{array}{l}\text { Endo et al. (2008) } \\
\text { (Japan) (Niigata) }\end{array}$ & Asia & 60 & $30 / 30$ & Digital caliper $(0,01 \mathrm{~mm})$ & $91.01(1.91)$ & $\begin{array}{l}77.48 \\
(2.17)\end{array}$ & $\begin{array}{c}91.14(2.09) / \\
90.88(2.20)\end{array}$ & $\begin{array}{l}77.63(1.82) / \\
77.33(2.49)\end{array}$ & - & Yes \\
\hline $\begin{array}{l}\text { Al Sulaimani et al. } \\
\text { (2006) (Saudi Arabia) } \\
\text { (Jeddah) }\end{array}$ & Asia & 98 & $62 / 36$ & Ortho-l software & $93.90(4.07)$ & $\begin{array}{l}81.11 \\
(5.07)\end{array}$ & NS & NS & & Yes \\
\hline $\begin{array}{l}\text { Ciger et al. (2006) } \\
\text { (Turkey) (Hacettepe) }\end{array}$ & Europe & 125 & $70 / 55$ & Digital caliper $(0,01 \mathrm{~mm})$ & $91.95(2.20)$ & $\begin{array}{l}77.95 \\
(2.35)\end{array}$ & $\begin{array}{l}91.97(1.65) / \\
91.82(1.99)\end{array}$ & $\begin{array}{l}78.62(2.24) / \\
78.43(2.41)\end{array}$ & - & Yes \\
\hline
\end{tabular}


Peer-reviewed version available at Journal of Orthodontics 2019; doi:10.1177/1465312519886322

\begin{tabular}{|c|c|c|c|c|c|c|c|c|c|c|}
\hline $\begin{array}{l}\text { Carreiro et al. (2005) } \\
\text { (Brazil) (Panamá) }\end{array}$ & America & 44 & $22 / 22$ & Microscribe 3DX & $92.13(2.08)$ & $\begin{array}{l}77.13 \\
(3.15)\end{array}$ & NS & NS & & Yes \\
\hline $\begin{array}{l}\text { Uysal et al. (2005) } \\
\text { (Turkey) (Konya) }\end{array}$ & Europe & 156 & $150 / 6$ & Digital caliper $(0,01 \mathrm{~mm})$ & $91.90(3.21)$ & $\begin{array}{l}78.56 \\
(3.23)\end{array}$ & $\begin{array}{l}91.65(3.51) / \\
91.57(2.98)\end{array}$ & $\begin{array}{l}78.18(3.31) / \\
78.44(3.18)\end{array}$ & - & Yes \\
\hline $\begin{array}{l}\text { Laino et al. (2004) } \\
\text { (Italy) (Campania) }\end{array}$ & Europe & 57 & $31 / 26$ & Digital caliper $(0,01 \mathrm{~mm})$ & $91.72(2.20)$ & $\begin{array}{l}78.12 \\
(2.41)\end{array}$ & NS & NS & - & Yes \\
\hline $\begin{array}{l}\text { Araújo et al. (2003) } \\
\text { (Brazil) (Belo } \\
\text { Horizonte) }\end{array}$ & America & 100 & $58 / 42$ & Digital caliper $(0,01 \mathrm{~mm})$ & NS & $\begin{array}{l}78.18 \\
(2.85)\end{array}$ & NS & NS & - & Yes \\
\hline $\begin{array}{l}\text { Alkofide et al. (2002) } \\
\text { (Saudi Arabia) } \\
\text { (Jeddah) }\end{array}$ & Asia & 60 & $30 / 30$ & Digital caliper $(0,01 \mathrm{~mm})$ & $92.24(2.04)$ & $\begin{array}{l}78.77 \\
(2.74)\end{array}$ & $\begin{array}{l}92.12(1.67) / \\
92.36(2.37)\end{array}$ & $\begin{array}{c}78.75(2.27) / \\
78.79(3.19)\end{array}$ & - & Yes \\
\hline $\begin{array}{l}\text { Ta et al. (2001) } \\
\text { (Hong Kong) } \\
\text { (Sheung Wan) }\end{array}$ & Asia & 50 & $25 / 25$ & Digital caliper $(0,01 \mathrm{~mm})$ & $90.65(1.19)$ & $\begin{array}{l}77.55 \\
(1.80)\end{array}$ & $\begin{array}{c}91.10(1.00) / \\
90.20(1.20)\end{array}$ & $\begin{array}{c}77.60(1.80) / \\
77.50(1.80)\end{array}$ & - & Yes \\
\hline $\begin{array}{l}\text { Nie et al. (1999) } \\
\text { (China) (Beijing) }\end{array}$ & Asia & 60 & $30 / 30$ & Software $(0,01 \mathrm{~mm})$ & $93.27(2.48)$ & $\begin{array}{l}81.52 \\
(2.82)\end{array}$ & $\begin{array}{l}93.62(2.42) / \\
93.41(2.53)\end{array}$ & $\begin{array}{l}81.87(2.51) / \\
81.25(2.87)\end{array}$ & - & Yes \\
\hline \multirow[t]{2}{*}{$\begin{array}{l}\text { Crosby et al. (1989) } \\
\text { (USA) (Texas) }\end{array}$} & America & 30 & NS & Digital caliper $(0,01 \mathrm{~mm})$ & $91.30(2.40)$ & $\begin{array}{c}77.2 \\
(2.70)\end{array}$ & NS & NS & - & Yes \\
\hline & & & & Class II & & & & & & \\
\hline $\begin{array}{l}\text { Machado et al. (2018) } \\
\text { (Portugal) (Almada) }\end{array}$ & Europe & 51 & $36 / 15$ & Digital caliper $(0,01 \mathrm{~mm})$ & $91.51(2.69)$ & $\begin{array}{l}78.6 \\
(3.59)\end{array}$ & $\begin{array}{l}91.49(2.11) / \\
91.57(3.01)\end{array}$ & $\begin{array}{l}77.96(3.58) / \\
78.93(3.64)\end{array}$ & - & Yes \\
\hline $\begin{array}{l}\text { Saritha et al. (2017) } \\
\text { (India) (Telangana) }\end{array}$ & Asia & 103 & $70 / 33$ & Digital caliper $(0,01 \mathrm{~mm})$ & $92.296(1.997)$ & $\begin{array}{l}78.642 \\
(2.868)\end{array}$ & $\begin{array}{l}92.14(1.9) / \\
92.37(2.05)\end{array}$ & $\begin{array}{c}78.21(2.56) / \\
78.84(2.99)\end{array}$ & - & Yes \\
\hline $\begin{array}{l}\text { Mahmoud et al. } \\
\text { (2017) (Sudan) } \\
\text { (Khartoum) }\end{array}$ & Asia & 44 & NS & Digital caliper $(0,05 \mathrm{~mm})$ & $90.85(2.64)$ & $\begin{array}{l}78.14 \\
(4.35)\end{array}$ & NS & NS & - & Yes \\
\hline $\begin{array}{l}\text { Cançado et al. (2016) } \\
\text { (Brazil) (Dourados) }\end{array}$ & America & 324 & NS & Digital caliper $(0,01 \mathrm{~mm})$ & $91.46(2.06)$ & $\begin{array}{l}78.31 \\
(2.39)\end{array}$ & NS & NS & - & Yes \\
\hline $\begin{array}{l}\text { Al-Duliamy Iraq et } \\
\text { al. (2016) (Iraq) } \\
\text { (Baghdad) }\end{array}$ & Asia & 40 & NS & Digital caliper $(0,01 \mathrm{~mm})$ & $91.54(2.66)$ & $\begin{array}{l}79.05 \\
(2.64)\end{array}$ & NS & NS & - & Yes \\
\hline $\begin{array}{l}\text { Al-Duliamy Egypt et } \\
\text { al. (2016) (Egypt) } \\
\text { (Cairo) }\end{array}$ & Africa & 40 & NS & Digital caliper $(0,01 \mathrm{~mm})$ & $89.14(5.13)$ & $\begin{array}{l}78.46 \\
(3.97)\end{array}$ & NS & NS & - & Yes \\
\hline $\begin{array}{l}\text { Shastri et al. (2015) } \\
\text { (India (North)) } \\
\text { (Lucknow) }\end{array}$ & Asia & 50 & NS & Digital caliper & $90.77(2.13)$ & $\begin{array}{c}81.1 \\
(5.01)\end{array}$ & NS & NS & - & Yes \\
\hline $\begin{array}{l}\text { Bughaighis et al. } \\
\text { (2015) (Libya) } \\
\text { (Benghazi) }\end{array}$ & Africa & 85 & NS & Digital caliper $(0,01 \mathrm{~mm})$ & $91.5(2.43)$ & $\begin{array}{l}78.1 \\
(2.73)\end{array}$ & NS & NS & - & Yes \\
\hline $\begin{array}{l}\text { Ismail et al. (2015) } \\
\text { (Sudan) (Khartoum) }\end{array}$ & Africa & 59 & $27 / 22$ & Digital caliper $(0,1 \mathrm{~mm})$ & $92.05(3.11)$ & $\begin{array}{l}77.45 \\
(4.8)\end{array}$ & $\begin{array}{l}92.22(3.84) / \\
91.92(2.35)\end{array}$ & $\begin{array}{l}77.17(6.05) / \\
77.68(3.44)\end{array}$ & - & Yes \\
\hline $\begin{array}{l}\text { Maurya et al. (2015) } \\
\text { (India) (Madhya } \\
\text { Pradesh) }\end{array}$ & Asia & 60 & $30 / 30$ & Digital caliper $(0,01 \mathrm{~mm})$ & $91.69(2.4)$ & $\begin{array}{l}79.06 \\
(2.56)\end{array}$ & $\begin{array}{l}91.67(1.92) / \\
91.7(2.87)\end{array}$ & $\begin{array}{c}77.98(1.95) / \\
80.14(2.7)\end{array}$ & - & Yes \\
\hline $\begin{array}{l}\text { Zerouaoui et al. } \\
\text { (2014) (Morocco) }\end{array}$ & Africa & 30 & NS & Digital caliper & $92.597(2.41398)$ & $\begin{array}{c}79.5975 \\
(2.94213)\end{array}$ & NS & NS & - & Yes \\
\hline
\end{tabular}


(Rabat) (2014) (Ireland) (Dublin)

Asma et al. (2013)

(Malaysia) (Selangor)

Ali Hyder et al.

(2012) (Bangladesh)$$
\text { (Dhaka) }
$$

Kansal et al. (2012)

(India) (Karnataka)

O'Mahony et al.

(2011) (Ireland) (Cork)

Endo et al. (2010)

(Japan) (Niigata)

Oktay et al. (2010)

(Turkey) (Erzurum)

Strujić et al. (2009)

(Croatia) (Zagreb)

Endo et al. (2009)

(Japan) (Niigata)

Endo et al. (2008)

(Japan) (Niigata)

Al Sulaimani et al.

(2006) (Saudi Arabia) (Jeddah)

Uysal et al. (2005)

(Turkey) (Konya)

Laino et al. (2004)
(Italy) (Campania)

(Italy) (Campania)

Araújo et al. (2003)

(Brazil) (Belo

Horizonte)

Alkofide et al. (2002)

(Saudi Arabia)

(Jeddah)

Ta et al. (2001)

(Hong Kong)

(Sheung Wan)

(Chin) (Beijing)

Crosby et al. (1989)

Crosby et al. (1989)
(USA) (Texas)

Machado et al. (2018) (Portugal) (Almada)
Europe

Asia

Asia

Asia

Europe

Asia

Europe

Europe

Asia

Asia

Asia

Europe

Europe

America

Asia

Asia

Asia

America
$30 / 30$

$$
\text { Software }(0,01 \mathrm{~mm})
$$

Digital caliper $(0,01 \mathrm{~mm})$

20 / 20 Digital caliper $(0,01 \mathrm{~mm})$

Digital caliper $(0,01 \mathrm{~mm})$

$60 / 60$ OrhoAnalyzer (Software)

33 / 33 Digital caliper $(0,01 \mathrm{~mm})$

124 / 76 RMI 550 3D (0,01 mm)

60 / 49 Digital caliper $(0,01 \mathrm{~mm})$

42 / 36 Digital caliper $(0,01 \mathrm{~mm})$

$30 / 30 \quad$ Digital caliper $(0,01 \mathrm{~mm})$

$34 / 18$

Ortho-1 software

105 / 86 Digital caliper (0,01 mm)

18 / 6 Digital caliper $(0,01 \mathrm{~mm})$

48 / 52 Digital caliper $(0,01 \mathrm{~mm})$

$60 / 60$ Digital caliper (0,01 mm)

15 / 15 Digital caliper (0,01 mm)

$60 / 60 \quad$ Software $(0,01 \mathrm{~mm})$

NS

Digital caliper $(0,01 \mathrm{~mm})$

Class II - Division 1

$16 / 7$ Digital caliper $(0,01 \mathrm{~mm})$

$\begin{array}{ccc}90.2(2.27) & 76.5 & 89.9(2.31) / \\ & (2.77) & 90.5(2.24) \\ \text { NS } & 79.54 & \text { NS } \\ & (4.37) & \\ 90.9(2.79) & 78.5 & 91.31(2.25) / \\ & (3.93) & 90.56(3.25) \\ 91.52(3.37) & 79.1 & \text { NS } \\ & (3.94) & \\ 92.2(2.19) & 79.4 & 92.3(2.31) / \\ & (3.36) & 92.05(2.03) \\ 91.38(1.88) & \text { NS } & 91.48(1.91) / \\ & 78.67 & 92.28(1.87) \\ 92.06(2.16) & (2.53) & 91.9(2.18) / \\ 91.14(2.14) & 77.73 & \text { NS } \\ & (2.42) & 91.47(1.91) / \\ 91.57(2.34) & 77.68 & 91.66(2.65) \\ & (2.38) & 91.43(1.98) / \\ 91.3(1.94) & 77.93 & \\ & (2.25) & 91.17(1.91) \\ 93.06(3.65) & 81.88 & \text { NS } \\ & (4.31) & \\ 91.27(3.35) & 78.59 & \text { NS } \\ 91.24(1.85) & (3.48) & \\ & 78.04 & \text { NS } \\ \text { NS } & 7.35) & \\ & 78.16 & \text { NS } \\ & (2.21) & \end{array}$

$76.5(2.87) /$

$76.55(2.7)$

NS

$78.7(3.88) /$

78.37 (4.08)

$$
\text { NS }
$$

$79.5(3.56) /$

$79.35(3.2)$

$$
\text { NS }
$$

$78.43(2.46) /$ $78.48(2.58)$

NS

$78.22(2.25) /$

$78.07(2.41)$

$77.92(2.26) /$

$77.93(2.29)$

NS

NS

NS

NS

$92.5(2.17) / \quad 78.56(2.73) /$

$93.1(2.23)$

78.84 (2.17)

$77.8(1.7) / 77.7$

(1.4)

$90.4(0.7)$

$80.69(3.72) /$

$92.1(2.66) /$

$92.02(2.33)$

$80.89(2.54)$

NS

$91.5(2.56)$

77.51
$(3.9)$

NS

$91.4(2.8)$

$78.6(3.8)$

$90.5(1.8) /$

$91.9(3.1)$
Yes

Yes

Yes

Yes

Yes

Yes

Yes

Yes

Yes

Yes

Yes

Yes

Yes

Yes

Yes

Yes

Yes

Yes

Yes 
Peer-reviewed version available at Journal of Orthodontics 2019; doi:10.1177/1465312519886322

Mahmoud et al.
(2017) (Sudan)
(Khartoum)
Elsheikhi et al.
(2017) (Libya)
(Benghazi)
Chugh et al. (2015)
(India) (Lucknow)
Bughaighis et al.
(2015) (Libya)
(Benghazi)
Asma et al. (2013)
(Malaysia) (Selangor)
Ricci et al. (2013)
(Brazil) (São Paulo)
Kansal et al. (2012)
(India) (Karnataka)
O'Mahony et al.
(2011) (Ireland)
(Cork)
Oktay et al. (2010)
(Turkey) (Erzurum)
Ciger et al. (2006)
(Turkey) (Hacettepe)
Carreiro et al. (2005)
(Brazil) (Panamá)
Uysal et al. (2005)
(Turkey) (Konya)
Nie et al. (1999)
(China) (Beijing)
Crosby et al. (1989)
(USA) (Texas)

Machado et al. (2018)
(Portugal) (Almada)
Mahmoud et al.
(2017) (Sudan)
(Khartoum)
Bughaighis et al.
(2015) (Libya)
(Benghazi)
Asma et al. (2013)
(Malaysia) (Selangor)
Kansal et al. (2012)
(India) (Karnataka)
O'Mahony et al.
(2011) (Ireland)
(Cork)

Asi

Africa

Asia

Africa

Asia

America

Asia

Europe

Europe

Europe

America

Europe

America

Europe

Asia

Africa

Asia

Asia

Europe

(Cork)

41
20
40
73
50
35
237
60
100
71
54
157
60
30

NS Digital caliper $(0,01 \mathrm{~mm})$

$90.73(2.63)$

$10 / 10 \quad$ Digital caliper $(0,01 \mathrm{~mm}) \quad 91.38(3.06) \quad 76.29$

$20 / 20 \quad$ Digital caliper $(0,01 \mathrm{~mm}) \quad 91.53(2.49) \quad \begin{aligned} & 78.96 \\ & (3.56)\end{aligned}$

$91.53(2.49)$

78.96
$(3.56)$

NS

Digital caliper $(0,01 \mathrm{~mm})$

$91.49(2.58)$

78.08

NS Digital caliper $(0,01 \mathrm{~mm})$

NS

NS Digital caliper $(0,01 \mathrm{~mm})$

$90.67(2.4)$

78.75

NS Digital caliper $(0,01 \mathrm{~mm})$

$91.5(3.4)$

$30 / 30 \quad$ OrhoAnalyzer (Software)

$91.8(2.1)$

$61 / 39$ RMI $5503 \mathrm{D}(0,01 \mathrm{~mm})$

$91.86(2.07)$

$40 / 31 \quad$ Digital caliper $(0,01 \mathrm{~mm})$

90.83 (3.9)

26/28 Microscrrib 3DX

$92.24(2.56)$

$82 / 75$

Digital caliper $(0,01 \mathrm{~mm})$

$91.12(3.34)$

$92.16(2.5)$

Software $(0,01 \mathrm{~mm})$

$91.7(2.3)$

Digital caliper $(0,01 \mathrm{~mm})$

Class II - Division 2

Softwares $(0,01 \mathrm{~mm})$

$91.6(2.6)$

$78.6(3.4)$

Softwares $(0,01 \mathrm{~mm})$

$92.42(2.17)$

78.57

(1.53)

$91.56(1.21)$

78.2
$(2.29)$

Softwares $(0,01 \mathrm{~mm})$

NS

80.33

Softwares $(0,01 \mathrm{~mm})$

$91.8(2.9)$

(4.71)

Softwares $(0,01 \mathrm{~mm})$

$79.1(3)$

$92.6(2.2)$

$80.2(3)$

$30 / 30 \quad$ OrhoAnalyzer (Software)

NS

NS

$92.24(2.43) /$

$90.83(2.41)$

NS

NS

$90.37(2.35) /$

$90.76(2.45)$

NS

$91.8(2.4) /$

$91.8(1.8)$

$92.22(2.05) /$

$91.64(2.07)$

91.05 (4.24)

NS

$91.19(2.53) /$

$91.07(3.96)$

$92.11(2.61)$

NS

$92.3(2) / 91.3$

(2.9)

NS

NS

NS

NS

$92.8(2.1) /$

$92.3(2.2)$
NS

NS

$79.95(2.78) /$

77.97 (3.66)

NS

NS

$77.27(2.08) /$

77.3 (2.65)

NS

$77.9(3.6) / 79.3$

(3.3)

78.1 (2.17)/

$78.58(2.46)$

$77.94(2.46)$

78.11 (2.65)

NS

$78.68(3.06) /$

$78.33(2.42)$

$80.31(3.87) /$

$80.8(2.42)$

NS

$78.5(3.7) / 78.7$

(3.3)

NS

NS

NS

NS

$81.1(2.7) / 79.4$

(3.1)
Yes

Yes

Yes

Yes

Yes

Yes

Yes

Yes

Yes

Yes

Yes

Yes

Yes

Yes

Yes

Yes

Yes

Yes

Yes

Yes 
Peer-reviewed version available at Journal of Orthodontics 2019; doi:10.1177/1465312519886322

\begin{tabular}{|c|c|c|c|c|c|c|c|c|c|c|}
\hline $\begin{array}{l}\text { Oktay et al. (2010) } \\
\text { (Turkey) (Erzurum) }\end{array}$ & Europe & 100 & $63 / 37$ & RMI 550 3D (0,01 mm) & $92.26(2.22)$ & $\begin{array}{l}78.98 \\
(2.67)\end{array}$ & $\begin{array}{c}92.42(2.15) / \\
92.16(2.26)\end{array}$ & $\begin{array}{l}78.76(2.67) / \\
78.38(2.69)\end{array}$ & - & Yes \\
\hline $\begin{array}{l}\text { Uysal et al. (2005) } \\
\text { (Turkey) (Konya) }\end{array}$ & Europe & 34 & $23 / 11$ & Softwares $(0,01 \mathrm{~mm})$ & $91.94(3.34)$ & $79(4.23)$ & $\begin{array}{l}90.81(2.27) / \\
89.81(4.65)\end{array}$ & $\begin{array}{c}79.63(3.35) / \\
78.7(4.64)\end{array}$ & - & Yes \\
\hline $\begin{array}{l}\text { Nie et al. (1999) } \\
\text { (China) (Beijing) }\end{array}$ & Asia & 60 & $30 / 30$ & Software $(0,01 \mathrm{~mm})$ & $91.95(2.47)$ & $\begin{array}{c}81.02 \\
(43376)\end{array}$ & $\begin{array}{l}92.09(2.7) / \\
91.82(2.26)\end{array}$ & $\begin{array}{l}81.07(3.52) / \\
80.97(2.66)\end{array}$ & - & Yes \\
\hline $\begin{array}{l}\text { Crosby et al. (1989) } \\
\text { (USA) (Texas) }\end{array}$ & America & 29 & NS & Softwares $(0,01 \mathrm{~mm})$ & $91.5(3.1)$ & $76.8(5.3)$ & NS & NS & - & Yes \\
\hline \multicolumn{11}{|c|}{ Class III } \\
\hline $\begin{array}{l}\text { Machado et al. (2018) } \\
\text { (Portugal) (Almada) }\end{array}$ & Europe & 38 & $25 / 13$ & Softwares $(0,01 \mathrm{~mm})$ & $92(2)$ & $78(2.9)$ & $\begin{array}{l}91.8(1.6) / \\
92.1(2.3)\end{array}$ & $\begin{array}{c}78(2.9) / 78.1 \\
(2.9)\end{array}$ & - & Yes \\
\hline $\begin{array}{l}\text { Saritha et al. (2017) } \\
\text { (India) (Telangana) }\end{array}$ & Asia & 40 & $21 / 19$ & Softwares $(0,01 \mathrm{~mm})$ & $92.967(1.546)$ & $\begin{array}{l}79.72 \\
(2.52)\end{array}$ & $\begin{array}{l}92.99(1.75) / \\
92.94(1.38)\end{array}$ & $\begin{array}{l}79.92(3.06) / \\
79.54(1.97)\end{array}$ & - & Yes \\
\hline $\begin{array}{l}\text { Mahmoud et al. } \\
\text { (2017) (Sudan) } \\
\text { (Khartoum) }\end{array}$ & Asia & 11 & NS & Softwares $(0,01 \mathrm{~mm})$ & $91.38(2.04)$ & $\begin{array}{l}78.37 \\
(3.16)\end{array}$ & NS & NS & - & Yes \\
\hline $\begin{array}{l}\text { Elsheikhi et al. } \\
\text { (2017) (Libya) } \\
\text { (Benghazi) }\end{array}$ & Africa & 20 & $10 / 10$ & Softwares $(0,01 \mathrm{~mm})$ & $92.05(2.96)$ & $\begin{array}{l}76.65 \\
(4.09)\end{array}$ & NS & NS & - & Yes \\
\hline $\begin{array}{l}\text { Cançado et al. (2016) } \\
\text { (Brazil) (Dourados) }\end{array}$ & America & 66 & NS & Softwares $(0,01 \mathrm{~mm})$ & $91.22(2.07)$ & $\begin{array}{l}77.9 \\
(2.85)\end{array}$ & NS & NS & - & Yes \\
\hline $\begin{array}{l}\text { Al-Duliamy et al. } \\
\text { (2016) (Iraq) } \\
\text { (Baghdad) }\end{array}$ & Asia & 10 & NS & Softwares $(0,01 \mathrm{~mm})$ & $91.82(2.24)$ & $\begin{array}{l}78.8 \\
(2.15)\end{array}$ & NS & NS & - & Yes \\
\hline $\begin{array}{l}\text { Al-Duliamy et al. } \\
\text { (2016) (Egypt) } \\
\text { (Cairo) }\end{array}$ & Africa & 10 & NS & Softwares $(0,01 \mathrm{~mm})$ & $90.65(3.71)$ & $\begin{array}{r}78.65 \\
(4.2)\end{array}$ & NS & NS & - & Yes \\
\hline $\begin{array}{l}\text { Chugh et al. (2015) } \\
\text { (India) (Lucknow) }\end{array}$ & Asia & 30 & NS & Softwares $(0,01 \mathrm{~mm})$ & $94.05(2.01)$ & $\begin{array}{l}81.23 \\
(3.11)\end{array}$ & $\begin{array}{l}94.48(1.83) / \\
93.47(2.11)\end{array}$ & $\begin{array}{l}81.96(3.17) / \\
80.49(2.98)\end{array}$ & - & Yes \\
\hline $\begin{array}{l}\text { Shastri et al. (2015) } \\
\text { (India (North)) } \\
\text { (Lucknow) }\end{array}$ & Asia & 20 & NS & Softwares $(0,01 \mathrm{~mm})$ & $91.33(2.32)$ & $\begin{array}{l}77.51 \\
(5.64)\end{array}$ & NS & NS & - & Yes \\
\hline $\begin{array}{l}\text { Bughaighis et al. } \\
\text { (2015) (Libya) } \\
\text { (Benghazi) }\end{array}$ & Africa & 13 & NS & Softwares $(0,01 \mathrm{~mm})$ & $90.97(2.93)$ & $\begin{array}{l}77.48 \\
(3.51)\end{array}$ & NS & NS & - & Yes \\
\hline $\begin{array}{l}\text { Ismail et al. (2015) } \\
\text { (Sudan) (Khartoum) }\end{array}$ & Africa & 43 & $27 / 16$ & Softwares $(0,01 \mathrm{~mm})$ & $92.6(3.01)$ & $\begin{array}{l}77.71 \\
(4.2)\end{array}$ & $\begin{array}{l}93.58(2.71) / \\
92.02(3.03)\end{array}$ & $\begin{array}{l}78.01(4.12) / \\
77.53(4.24)\end{array}$ & - & Yes \\
\hline $\begin{array}{l}\text { Maurya et al. (2015) } \\
\text { (India) (Madhya } \\
\text { Pradesh) }\end{array}$ & Asia & 24 & $12 / 12$ & Softwares $(0,01 \mathrm{~mm})$ & $94.72(1.13)$ & $\begin{array}{l}84.49 \\
(1.33)\end{array}$ & $\begin{array}{l}95.51(0.72) / \\
93.93(0.88)\end{array}$ & $\begin{array}{c}85.56(0.93) / \\
84.33(1.57)\end{array}$ & - & Yes \\
\hline $\begin{array}{c}\text { Zerouaoui et al. } \\
\text { (2014) (Morocco) } \\
\text { (Rabat) }\end{array}$ & Africa & 30 & NS & Softwares $(0,01 \mathrm{~mm})$ & $92.075(2.2062)$ & $\begin{array}{c}78.2358 \\
(2.85751)\end{array}$ & NS & NS & - & Yes \\
\hline $\begin{array}{l}\text { McSwiney et al. } \\
\text { (2014) (Ireland) } \\
\text { (Dublin) }\end{array}$ & Europe & 60 & NS & Softwares $(0,01 \mathrm{~mm})$ & $92.25(2.31)$ & $\begin{array}{l}78.35 \\
(2.42)\end{array}$ & $\begin{array}{l}92.3(2.27) / \\
92.2(2.41)\end{array}$ & $\begin{array}{l}78.15(2.37) / \\
78.55(2.58)\end{array}$ & - & Yes \\
\hline $\begin{array}{l}\text { Asma et al. (2013) } \\
\text { (Malaysia) (Selangor) }\end{array}$ & Asia & 50 & NS & Softwares $(0,01 \mathrm{~mm})$ & NS & $\begin{array}{l}79.09 \\
(2.82)\end{array}$ & NS & NS & - & Yes \\
\hline
\end{tabular}


Peer-reviewed version available at Journal of Orthodontics 2019; doi:10.1177/1465312519886322

\begin{tabular}{|c|c|c|c|c|c|c|c|c|c|c|}
\hline $\begin{array}{l}\text { Ali Hyder et al. } \\
\text { (2012) (Bangladesh) } \\
\text { (Dhaka) }\end{array}$ & Asia & 40 & $20 / 20$ & Digital caliper $(0,01 \mathrm{~mm})$ & $91.4(2.58)$ & $\begin{array}{c}78.5 \\
(3.15)\end{array}$ & $\begin{array}{l}91.58(2.62) / \\
91.28(2.6)\end{array}$ & $\begin{array}{l}78.7(3.28) / \\
78.43(3.09)\end{array}$ & - & Yes \\
\hline $\begin{array}{l}\text { Kansal et al. (2012) } \\
\text { (India) (Karnataka) }\end{array}$ & Asia & 24 & NS & Digital caliper $(0,01 \mathrm{~mm})$ & $91.8(3.1)$ & $78.9(5.3)$ & NS & NS & - & Yes \\
\hline $\begin{array}{l}\text { O'Mahony et al. } \\
\text { (2011) (Ireland) } \\
\text { (Cork) }\end{array}$ & Europe & 60 & $30 / 30$ & OrhoAnalyzer (Software) & $92.8(2.2)$ & $79.9(3.1)$ & $\begin{array}{c}92.7(2.2) / \\
92.9(2.1)\end{array}$ & $\begin{array}{c}80.3(3) / 79.6 \\
\quad(3.2)\end{array}$ & - & Yes \\
\hline $\begin{array}{l}\text { Endo et al. (2010) } \\
\text { (Japan) (Niigata) }\end{array}$ & Asia & 66 & $33 / 33$ & Digital caliper $(0,01 \mathrm{~mm})$ & $91.56(1.89)$ & NS & $\begin{array}{l}91.27(1.56) / \\
91.85(2.16)\end{array}$ & () / () & - & Yes \\
\hline $\begin{array}{l}\text { Oktay et al. (2010) } \\
\text { (Turkey) (Erzurum) }\end{array}$ & Europe & 100 & $58 / 42$ & RMI 550 3D (0,01 mm) & $92.87(1.92)$ & $\begin{array}{l}79.3 \\
(2.94)\end{array}$ & $\begin{array}{l}92.81(2.05) / \\
92.92(1.83)\end{array}$ & $\begin{array}{l}79.39(3.13) / \\
79.24(2.83)\end{array}$ & - & Yes \\
\hline $\begin{array}{l}\text { Strujić et al. (2009) } \\
\text { (Croatia) (Zagreb) }\end{array}$ & Europe & 81 & $45 / 36$ & Digital caliper $(0,01 \mathrm{~mm})$ & $92.08(1.82)$ & $\begin{array}{l}78.23 \\
(2.82)\end{array}$ & NS & NS & - & Yes \\
\hline $\begin{array}{l}\text { Endo et al. (2009) } \\
\text { (Japan) (Niigata) }\end{array}$ & Asia & 71 & $35 / 36$ & Digital caliper $(0,01 \mathrm{~mm})$ & $91.54(1.86)$ & $\begin{array}{l}77.84 \\
(2.16)\end{array}$ & $\begin{array}{l}91.28(1.53) / \\
91.81(2.12)\end{array}$ & $\begin{array}{l}77.39(1.93) / \\
78.31(2.29)\end{array}$ & - & Yes \\
\hline $\begin{array}{l}\text { Endo et al. (2008) } \\
\text { (Japan) (Niigata) }\end{array}$ & Asia & 60 & $30 / 30$ & Digital caliper $(0,01 \mathrm{~mm})$ & $91.65(1.86)$ & $\begin{array}{l}77.87 \\
(2.18)\end{array}$ & $\begin{array}{l}91.46(1.46) / \\
91.83(2.2)\end{array}$ & $\begin{array}{l}77.54(1.92) / \\
78.2(2.4)\end{array}$ & - & Yes \\
\hline $\begin{array}{l}\text { Carreiro et al. (2005) } \\
\text { (Brazil) (Panamá) }\end{array}$ & America & 46 & $23 / 23$ & Microscribe 3DX & $92.3(2.69)$ & $\begin{array}{l}79.54 \\
(4.46)\end{array}$ & ()$/()$ & () / () & - & Yes \\
\hline $\begin{array}{l}\text { Uysal et al. (2005) } \\
\text { (Turkey) (Konya) }\end{array}$ & Europe & 113 & $55 / 58$ & Digital caliper $(0,01 \mathrm{~mm})$ & $91.69(3.66)$ & $\begin{array}{l}78.83 \\
(3.46)\end{array}$ & $\begin{array}{l}92,34(3,67) / \\
91,01(3,56)\end{array}$ & $\begin{array}{l}79,59(3,67) / \\
78,03(3,06)\end{array}$ & - & Yes \\
\hline $\begin{array}{l}\text { Al Sulaimani et al. } \\
\text { (2006) (Saudi Arabia) } \\
\text { (Jeddah) }\end{array}$ & Asia & 10 & $2 / 8$ & Ortho-l software & $96.3(1.45)$ & $\begin{array}{l}80.58 \\
(3.74)\end{array}$ & NS & NS & - & Yes \\
\hline $\begin{array}{l}\text { Laino et al. (2004) } \\
\text { (Italy) (Campania) }\end{array}$ & Europe & 13 & $6 / 7$ & Digital caliper $(0,01 \mathrm{~mm})$ & $90.94(2.26)$ & $\begin{array}{l}78.19 \\
(2.27)\end{array}$ & NS & NS & - & Yes \\
\hline $\begin{array}{l}\text { Araújo et al. (2003) } \\
\text { (Brazil) (Belo } \\
\text { Horizonte) }\end{array}$ & America & 100 & $49 / 51$ & Digital caliper $(0,01 \mathrm{~mm})$ & NS & $\begin{array}{l}79.03 \\
(2.35)\end{array}$ & NS & NS & - & Yes \\
\hline $\begin{array}{l}\text { Alkofide et al. (2002) } \\
\text { (Saudi Arabia) } \\
\text { (Jeddah) }\end{array}$ & Asia & 60 & $60 / 60$ & Digital caliper $(0,01 \mathrm{~mm})$ & $92.71(2.12)$ & $\begin{array}{l}78.5 \\
(2.53)\end{array}$ & $\begin{array}{l}93.2(2.15) / \\
92.21(2.02)\end{array}$ & $\begin{array}{l}79.66(2.52) / \\
77.34(1.98)\end{array}$ & - & Yes \\
\hline $\begin{array}{l}\text { Ta et al. (2001) } \\
\text { (Hong Kong) } \\
\text { (Sheung Wan) }\end{array}$ & Asia & 30 & $15 / 15$ & Digital caliper $(0,01 \mathrm{~mm})$ & $91.45(1.8)$ & $\begin{array}{l}79.43 \\
(2.55)\end{array}$ & $\begin{array}{l}91.2(2.1) / \\
91.7(1.4)\end{array}$ & $\begin{array}{c}77.9(3.1) / 79.2 \\
(1.8)\end{array}$ & - & Yes \\
\hline $\begin{array}{l}\text { Nie et al. (1999) } \\
\text { (China) (Beijing) }\end{array}$ & Asia & 120 & $60 / 60$ & Software $(0,01 \mathrm{~mm})$ & $95.6(2.62)$ & $\begin{array}{l}82.74 \\
(2.76)\end{array}$ & $\begin{array}{l}95.68(2.78) / \\
95.52(2.44)\end{array}$ & $\begin{array}{l}82.6(2.94) / \\
82.88(2.56)\end{array}$ & - & Yes \\
\hline
\end{tabular}



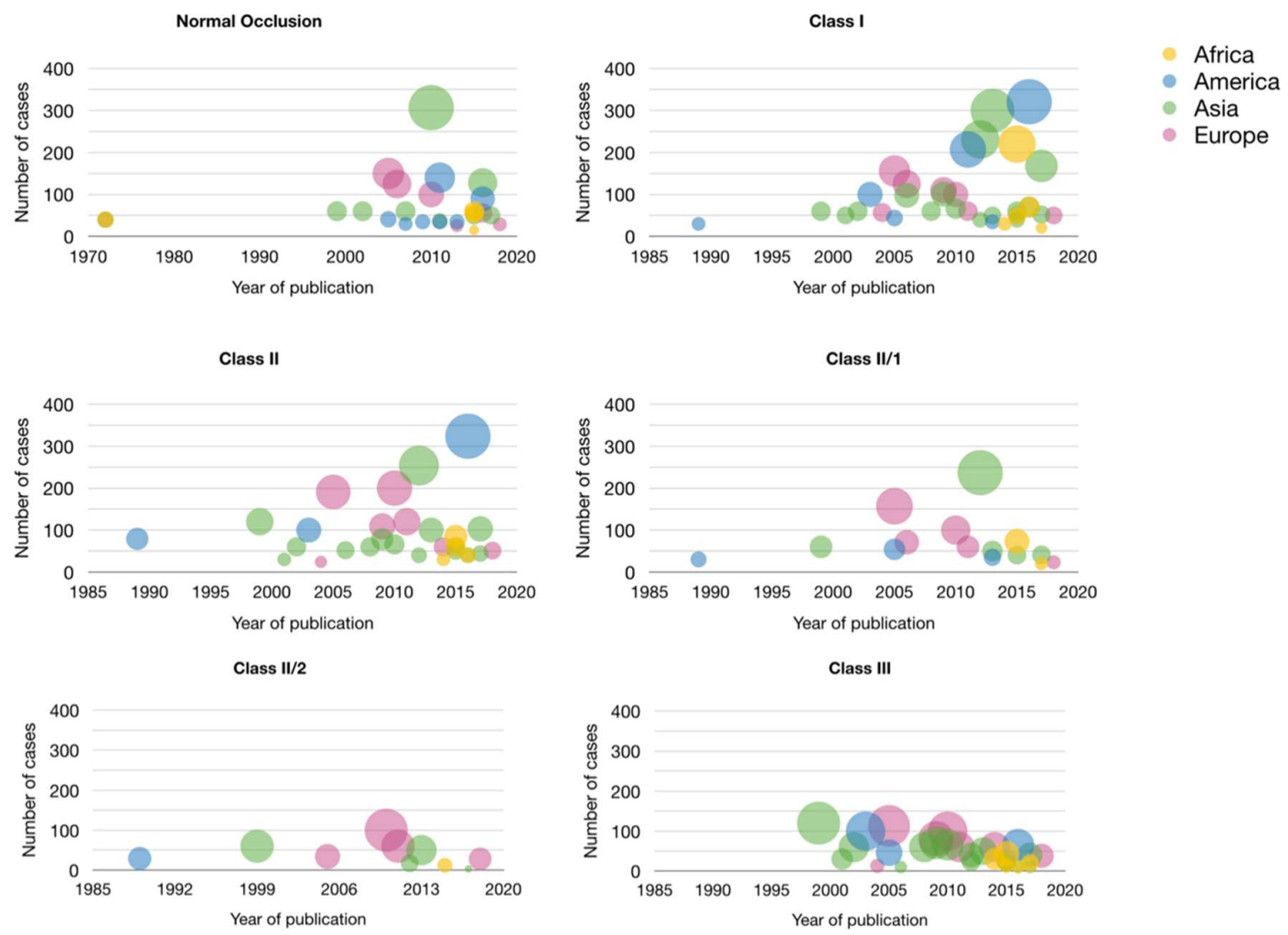

Figure 2: Datasets by year and population group by normal occlusion and Angle's malocclusion groups. Area of the circle is proportional to sample size.

\section{Risk of bias across studies}

Quality Assessment Tool for Observational Cohort and Cross-Sectional Studies statement proposed by the NIHLBI score in the final sample of articles ranged from 6 to 8 out of 10 (as shown in supplement Table S3). Among the included studies, no study presented high quality. Moreover, forty-seven articles presented medium quality, of which eleven articles presented 8 points $(30,33,37,43,44,48,52,53,58,67,75)$, and thirty-six articles presented 7 points $(12,22,25,26,31,34-36,38,39,42,45-51,54-57,59-65,67,68,70-72,76)$. Five were of low quality (77-81) and, consequently, were excluded.

Moreover, two articles were excluded $(82,83)$ due to abnormal standard deviation (SD) values (10 and 100 times lower than the mean value of standard deviation presented in the remaining studies, respectively). These unusual SD values frame narrow confidence intervals gaining unreasonable weight in the meta-analysis. We unsuccessfully tried to contact the authors, and therefore, we decided for the exclusion of these, pondering the likely negative consequences for the veracity of the results.

More specifically, only seven studies reported the setting, locations and relevant dates of cast models $(37,38,49,52,53,58,67)$, and seven determined the sample size $(30,33,43,44,48,58,75)$. Strategies to minimize the potential sources of bias were not clearly described in most articles. Nine fail to explain how they evaluated intra- and/or inter-examiner errors or random error determination $(38,49,77,79-84)$.

\section{Findings from Meta-analysis}

\section{Pairwise MA findings for Normal occlusion}

In normal occlusion group, the assessment of OR and AR was sourced from 24 and 25 studies, respectively (Figure 3 and 4). All those twenty-five studies provided data for AR assessment, while one study (61) had no data regarding OR. Global pooled results suggest an OR mean of $91.74 \%$ (95\% Cl: 91.37-92.10) and an AR mean 
of $78.24 \%$ (95\% Cl: 77.85-78.63). Globally, in both synthesis heterogeneity was high $\left(I^{2}=92.59 \%\right.$ and $I^{2}=90.99 \%$ in $O R$ and $A R$, respectively).

Next, we looked for gender differences on OR and AR at global level through gender mean difference metaanalysis. Only Class I presented a gender impact with male patients having higher $\mathrm{OR}(0.30,95 \% \mathrm{Cl} 0.00-0.59)$ and $\operatorname{AR}(0.41,95 \% \mathrm{Cl} 0.00-0.83)$ mean values than females (Supplement Table S4).

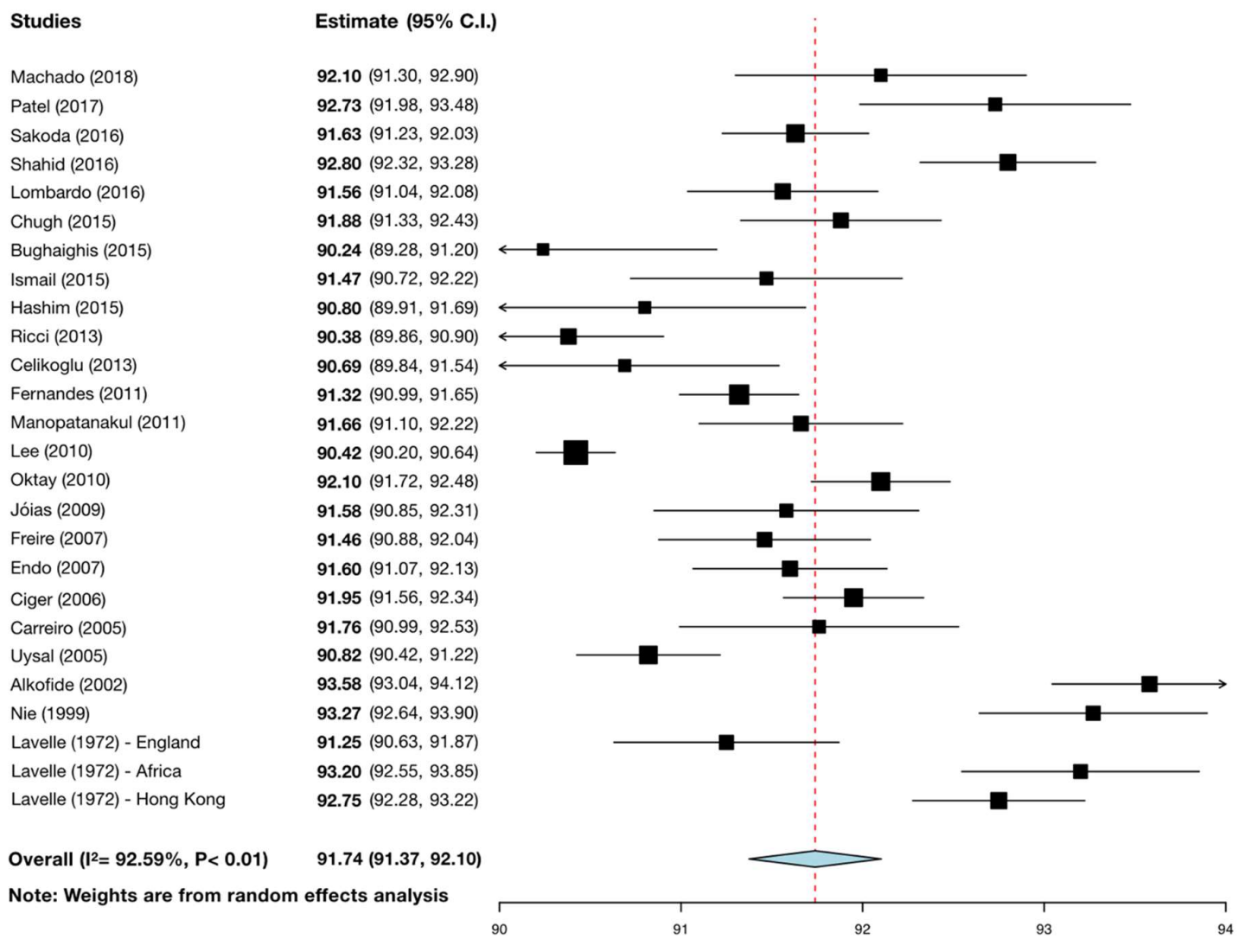

Figure 3: Forest plot of studies with OR mean values for normal occlusion patients. Mean effect size estimates have been calculated with $95 \%$ confidence intervals and are shown in the figure. Area of squares represents sample size, continuous horizontal lines and diamonds width represents $95 \%$ confidence interval. Blue diamond center and the vertical red dotted line point to the overall pooled estimate. 


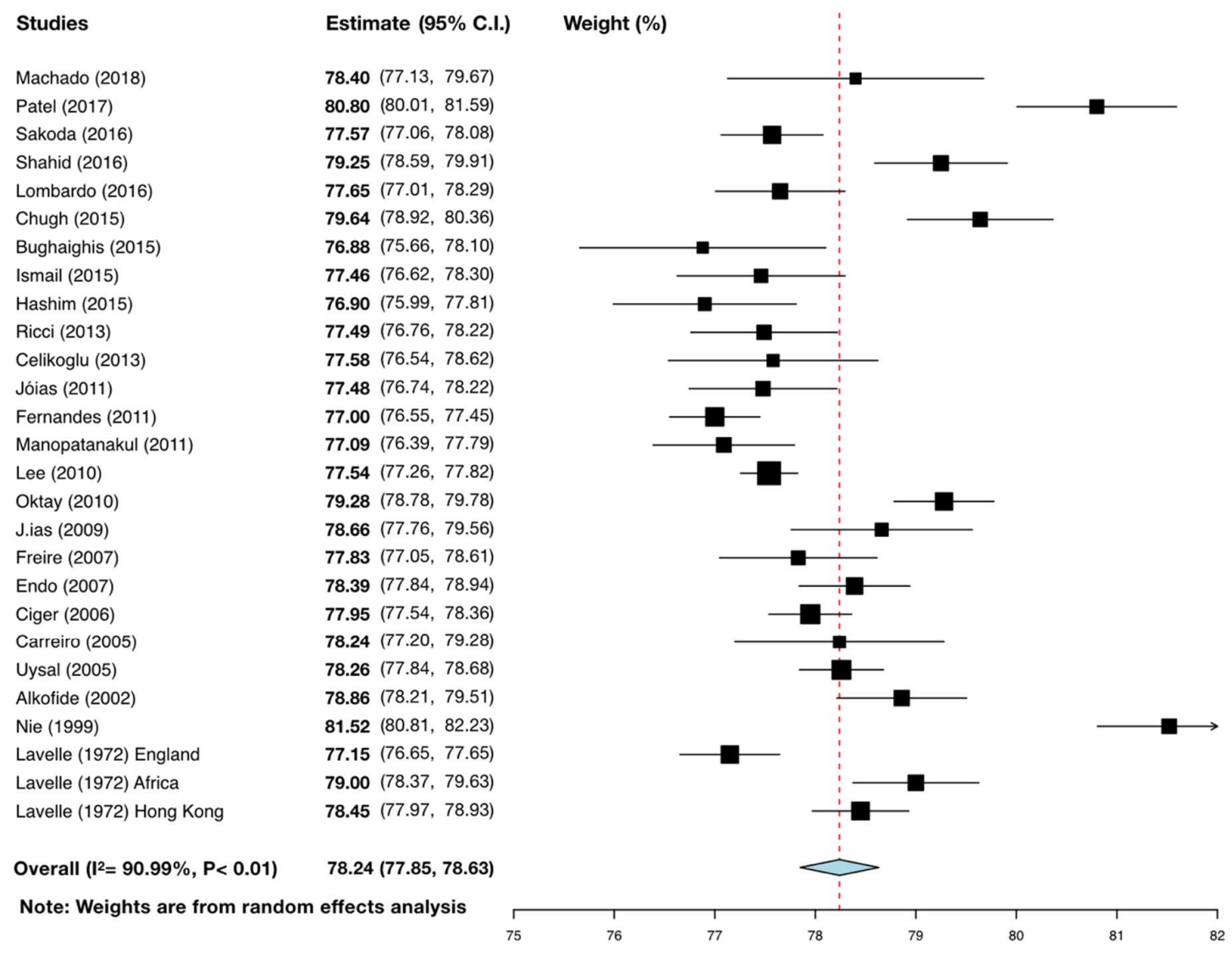

Figure 4: Forest plot of studies with AR mean values for normal occlusion patients. Mean effect size estimates have been calculated with $95 \%$ confidence intervals and are shown in the figure. Area of squares represents sample size, continuous horizontal lines and diamonds width represents $95 \%$ confidence interval. Blue diamond center and the vertical red dotted line point to the overall pooled estimate.

Pairwise MA Normal Occlusion vs. Bolton's original values

Direct comparison of the PMA pooled estimates for AR and OR normal occlusion mean values with Bolton's original values, through Z-test, revealed no significant differences, however, the statistical power for both analysis were extremely low, $5.8 \%$ and $5.7 \%$, respectively (Table 2 ).

Table 2: Comparison of Pairwise MA of normal occlusion with Bolton's original values.

\begin{tabular}{|c|c|c|c|c|c|c|c|c|c|c|}
\hline & \multicolumn{5}{|c|}{ AR } & \multicolumn{5}{|c|}{ OR } \\
\hline & $\mathbf{N}$ & $\begin{array}{c}\text { Mean } \\
(\%)\end{array}$ & $\begin{array}{l}\text { SD } \\
(\%)\end{array}$ & $\begin{array}{l}P(Z- \\
\text { test })\end{array}$ & $\begin{array}{c}\text { Statistical } \\
\text { Power }\end{array}$ & $\mathbf{N}$ & $\begin{array}{c}\text { Mean } \\
(\%)\end{array}$ & $\begin{array}{l}\text { SD } \\
(\%)\end{array}$ & $\begin{array}{l}P(Z- \\
\text { test) }\end{array}$ & $\begin{array}{c}\text { Statistical } \\
\text { Power }\end{array}$ \\
\hline $\begin{array}{l}\text { PMA Normal } \\
\text { occlusion }\end{array}$ & 1894 & 78.24 & 8.7 & \multirow{2}{*}{0.527} & \multirow{2}{*}{$5.8 \%$} & 1859 & 91.74 & 8.0 & \multirow{2}{*}{0.806} & \multirow{2}{*}{$5.7 \%$} \\
\hline $\begin{array}{c}\text { Bolton's Original } \\
\text { Values }\end{array}$ & 55 & 77.20 & 1.65 & & & 55 & 91.3 & 1.91 & & \\
\hline
\end{tabular}




\section{Bayesian Network meta-analysis findings for Angle's malocclusions groups}

The difference in mean change for normal occlusion (baseline) compared with different Angle's malocclusion groups is presented in table 3 , and can be seen as a measure of the average effort required to treat each represented malocclusion towards a proportional occlusion. In studies with no normal occlusion data, the comparison with the different types of Angle's malocclusions was not possible. Thus, we adopted a bayesian network meta-analysis approach to pool all available direct and indirect comparisons between normal occlusion versus Angle's Class I, Class II, Class II division 1, Class II division 2 and Class III values. The network fit statistic outcome included mean values for each Angle's malocclusions and the estimated normal versus malocclusion difference of means, with the degree of certainty of such differences reported as credibility intervals (Crl) (Table 3 ).

Table 3: Results of pairwise meta analysis of normal occlusion and Bayesian NMA of Angle's malocclusion groups.

\begin{tabular}{|c|c|c|c|c|c|c|c|c|c|c|}
\hline & \multicolumn{5}{|c|}{ AR } & \multicolumn{5}{|c|}{ OR } \\
\hline Pairwise MA & $\mathbf{N}$ & Mean & $\begin{array}{l}\text { Lower } \\
\text { Cl }\end{array}$ & $\begin{array}{l}\text { Upper } \\
\text { Cl }\end{array}$ & $\mathbf{I}^{2}$ & $\mathbf{N}$ & Mean & LowerCI & UpperCI & $\mathbf{I}^{2}$ \\
\hline Normal occlusion & 1894 & 78.24 & 77.85 & 78.63 & 90.99 & 1859 & 91.74 & 91.37 & 92.10 & 92.59 \\
\hline Bayesian NMA & $\mathbf{N}$ & Mean & $\begin{array}{c}\text { Lower } \\
\text { Crl }\end{array}$ & $\begin{array}{c}\text { Upper } \\
\text { Crl }\end{array}$ & & $\mathbf{N}$ & Mean & LowerCrl & UpperCrI & \\
\hline $\begin{array}{l}\text { Normal occlusion } \\
\text { (network adjusted) }\end{array}$ & 720 & 78.24 & 61.12 & 95.25 & & 720 & 91.75 & 76.21 & 107.37 & \\
\hline Class I & 2767 & 78.17 & 61.03 & 95.30 & & 2683 & 91.75 & 76.51 & 107.62 & \\
\hline Class II & 2429 & 78.12 & 61.02 & 95.18 & & 2563 & 91.99 & 76.25 & 107.40 & \\
\hline Class II / div 1 & 1001 & 78.06 & 60.99 & 95.12 & & 1051 & 91.813 & 76.06 & 107.11 & \\
\hline Class II / div 2 & 343 & 78.49 & 61.47 & 95.43 & & 343 & 91.57 & 76.38 & 107.33 & \\
\hline Class III & 1393 & 78.92 & 61.93 & 95.99 & & 1409 & 91.812 & 77.21 & 108.20 & \\
\hline
\end{tabular}

$\mathrm{N}$, total sample size; Mean; Cl, confidence interval; Crl, credibility interval; $\mathrm{Cl} / \mathrm{Crl}$ boundaries and $\mathrm{I} 2$ in \%

The mean change from normal occlusion for AR means Class I, Class II, Class II division 1 and Class II division 2 was -0.03 (95\% Crl, -0.29 to 0.23$),-0.10$ (95\% Crl, -0.37 to 0.16$),-0.16$ (95\% Crl, -0.45 to $0.14)$ and $0.25(95 \% \mathrm{Crl},-0.14$ to 0.64$)$, respectively, but in all, the $95 \% \mathrm{Crl}$ included zero. A similar trend was also observed for OR means when we compared the means of Class II, Class II division 1 and Class II division 2 with normal occlusion (Table 4).

In contrast, we found a meaningful difference between Angle's Class III versus normal occlusion both for OR and AR means $(0.89,95 \% \mathrm{Crl}, 0.66$ to 1.12 , and $0.66,95 \% \mathrm{Crl}, 0.38$ to 0.94 , respectively), and for Class I $(0.25,95 \% \mathrm{Crl}, 0.03$ to 0.47$)$ against normal occlusion AR means, since the null difference is not within the credibility region (Table 4). 
Table 4: Results of Bayesian Network Meta-Analysis for Angle's malocclusion groups.

\begin{tabular}{|c|c|c|c|c|c|c|c|c|}
\hline $\begin{array}{c}\text { Difference to Normal Occlusion } \\
(\%)\end{array}$ & \multicolumn{3}{|c|}{ AR } & \multicolumn{3}{c|}{ OR } \\
\hline Bayesian NMA & N & Mean & $\begin{array}{c}\text { Lower } \\
\text { Crl }\end{array}$ & $\begin{array}{c}\text { Upper } \\
\text { Crl }\end{array}$ & N & Mean & $\begin{array}{c}\text { Lower } \\
\text { Crl }\end{array}$ & $\begin{array}{c}\text { Upper } \\
\text { Crl }\end{array}$ \\
\hline Class I & & & & & & & & \\
\hline Class II & 2767 & -0.03 & -0.29 & 0.23 & 2683 & $\mathbf{0 . 2 5}$ & $\mathbf{0 . 0 3}$ & $\mathbf{0 . 4 7}$ \\
\hline Class II / div 1 & 2429 & -0.10 & -0.37 & 0.16 & 2563 & 0.06 & -0.17 & 0.25 \\
\hline Class II / div 2 & 1001 & -0.16 & -0.45 & 0.14 & 1051 & -0.16 & -0.41 & 0.09 \\
\hline Class III & 343 & 0.25 & -0.14 & 0.64 & 343 & 0.08 & -0.23 & 0.40 \\
\hline
\end{tabular}

Baseline normal occlusion is the covariate in the adjusted network meta-analysis.

$\mathrm{N}$, total sample size; Crl, credibility interval; Mean and Crls boundaries in \%. Bold means that the null difference in not within the credibility interval.

\section{Additional analyses}

Funnel plots revealed no evidence of publication bias (Supplement Figure S5).

\section{Discussion \\ Summary of Main Findings}

To the best of our knowledge, this is the first systematic review that attempted to estimate global OR and AR values in patients with normal occlusion and Angle's malocclusion. Despite the apparent gap in observational studies about normal occlusion between 1972 and 1998, the last 20 years have been of increased interest (Figure 2). Our results in normal occlusion patients demonstrated that, globally, the OR mean was $91.7 \%$ (95\% $\mathrm{Cl}$ : 91.4-92.1) and AR mean was $78.2 \%(95 \% \mathrm{Cl}$ : 77.9-78.6), while the values proposed by Bolton (1) were respectively smaller.

In fact, pooled PMA normal occlusion estimates were not significantly different from Bolton's values, however this direct comparison, though necessary, is quite unfair and disproportionate as shown by the extraordinarily low statistical power. Also, the computed standard deviations from meta-analytical pooled estimates revealed very discrepant and elevated values when compared with Bolton's ones, and we believe that these direct comparisons are biased since it is not adequate to compare so unequal samples.

It is important to remark that we have not made world subgroups based on the continent since the studies are not fully representative of the continent as a whole. There is a lack of studies in North America and Oceania continent. Similarly, the African continent is portrayed only by Libya, Egypt, Morocco and Sudan, the European continent is represented mainly by Turkey and Ireland investigations, the Asia continent is mostly represented by studies from India, and the American continent only had two study from North America and the remaining studies are from Brazil. Despite this restriction, future research should address race and genetic backgrounds to weigh their influence on the mesiodistal proportions since in this study it was not possible to perform due to the lack of such data. Still, globalization and miscegenation strongly support the concept of non-static proportions and the necessity for continued research. Further, gender and geographic location, in general, are not factors that influence dental width proportions. 
Regarding the relation between normal occlusion and Angle malocclusions, in general, our results determined no significant difference in the tooth size discrepancy existed for the OR and AR between normal occlusion and different malocclusion groups, except for the Class III malocclusion both in AR and OR, and Class I malocclusion only for OR. Under these circumstances, the results indicate that the discrepancy of intermaxillary tooth size may be one of the important factors in the cause of malocclusions, especially in Angle's Class III.

The results also suggest that these OR and AR differences for normal occlusion in Angle's Class I and III may be explained by upper or/and lower discrepancy. For both Angle's Class I and III difference for normal occlusion, a possible clinical explanation for this discrepancy may be due to smaller mesiodistal maxillary tooth sizes or/and greater mesiodistal mandibular widths.

\section{Quality of the Evidence and Potential Biases in the Review Process}

All studies included in meta-analysis presented overall medium quality, according to our pre-defined quality assessment and risk of bias. However, there are important matters that need to be pointed. A hypothetical limitation would be the fact that this systematic review only contains observational studies. However, except for restorative or traumatic reasons, teeth mesiodistal width remains prospectively unchanged. Therefore, RCTs, prospective or retrospective studies on this thematic, unless as the result of a secondary observation, would be inappropriate.

On the other hand, we have to emphasize that most studies lack sample size calculation and are nonrepresentative of the population, but rather from an academic setting. Besides, too many studies show a lack of information on calibration method or the number of examiners. These items are extremely important to minimize selection bias and strengthen the generalization of results, and its absence weakens the results of this systematic review. Additionally, no study has reported the existence of blinding examiners, since presumably the researchers themselves were involved in teeth measurements and Angle's evaluation. This potential bias should be considered in future research.

Significantly, the heterogeneity revealed by our meta-analysis refers, conceptually, to the variation in study outcomes between studies. This variation per se could flag some worrisome, however, we need to carefully assess this discrepancy, contrary to common meta-analysis. In our opinion, these results cannot be concluded as a high methodological variability rather than a high variableness of mesiodistal width proportions among the populations.

Regarding methodology, most studies took teeth measurements from plaster models. Only one investigation used intraoral 3-dimensional (3D) scanne(68), another used CBCT (75), three studies have digitized plaster models and subsequently performed the measurements $(22,57,60)$, and also three used an electronic measuring device $(52,64,70)$. Although in the past, calliper measurement in plaster models was the gold standard, nowadays the study of models with virtual 3D technology have higher reliability and accuracy (8588) and should be used as the first choice for diagnosis and treatment planning in Orthodontics, specifically to determine the width of the teeth. Additionally, study models produced by CBCT are far from being perfect for replacing digital models. Hence, in the future, with proper improvement, CBCT will ensure a multiplicity of analyzes from a single record (89). Furthermore, it is imperative that, in addition to the mesiodistal width, the labio-lingual and inclination data should be evaluated since they may also present great variability in populations. Thus, it is more desirable a 3D orthodontic diagnosis and treatment plan rather than a 2D assessment.

\section{Conclusions}

The results of this systematic review show that global pooled OR and AR mean values for normal occlusion patients are slightly above Bolton's original values. Class I, for OR mean values, and Class III, for both OR and $A R$, are proportionally larger than normal occlusion patients. Gender had no impact on teeth mesiodistal proportion.

\section{Implications for Clinical Practice and Research}


Despite being one of several measures used in orthodontic planning, the results of this systematic review suggest that Bolton's original values may be slightly underestimated as OR and AR global standard original. The use of inadequate standard measures for the dental proportion of each population can lead to diagnostic errors and could influence the patient's treatment outcome. Also, despite these AR and OR mean values were originally developed only for tooth width reduction (through interproximal stripping or extraction), several patients with mesiodistal disproportionality, mainly due to microdontia or agenesis in one or more teeth, require post-orthodontic rehabilitation treatments. Thus, in the future is imperative to establish normative data for different malocclusions and their impact proportion management during orthodontic treatment.

As a result, in the future, there is a clear need for further studies with more stringent methodologies with regard to sample size calculation, more representative population samples, explicit calibration methods to reduce risk bias, fostering the use of digital systems, and greater focus on the race of the population being studied combined with genetic background analysis of the patients. In addition, the question arises of the importance of orthodontic consensus to those who must establish normative data, since the results of this study point to a difference from the original Bolton's values. Still, the evolution of our species by the miscegenation due to globalization makes pressing the need for the continuous research on the human proportions, and, in this case, on the mesiodistal teeth proportion, since, apparently, these are not immutable.

In the forthcoming investigations, we believe it will be substantial to investigate the pre-orthodontic patient, in order to seek the ideal post-orthodontic position of each tooth. In this way, we will be able to know which tooth or teeth need mesiodistal intervention, so that we can achieve a normal occlusion with proper mesiodistal proportion respecting the Andrew's six keys.

\section{Conflict of interest}

Nothing to declare.

\section{List of abbreviations}

OR: overall ratio; AR anterior ratio; NIHLBI: National Heart, Lung, and Blood Institute; CBCT: cone beam computed tomography; 3D: 3-dimensional; 2D: 2-dimensional; PMA: pairwise meta-analysis; NMA: network meta analysis; RCT: randomized controlled trial; $\mathrm{Cl}$ : confidence interval; SD: standard deviation; Crl: credibility intervals.

\section{References}

1. Bolton W. Disharmony in tooth size and its relation to the analysis and treatment of malocclusion. Angle Orthod. 1958;28(3):113-30.

2. Kravitz ND, Kusnoto B, BeGole E, Obrez A, Agran B. How well does Invisalign work? A prospective clinical study evaluating the efficacy of tooth movement with Invisalign. Am J Orthod Dentofac Orthop [Internet]. 2009;135(1):27-35. Available from: http://dx.doi.org/10.1016/j.ajodo.2007.05.018

3. Lombardo L, Arreghini A, Ramina F, Ghislanzoni LTH, Siciliani G. Predictability of orthodontic movement with orthodontic aligners: a retrospective study. Prog Orthod. 2017;18(1):1-12.

4. Bonwill W. The scientific articulation of the human teeth as founded on geometrical, mathematical and mechanical laws. Dent Items Interest. 1899;1(1):617-43.

5. Black G. Descriptive anatomy of human teeth. 4th ed. Philadelphia; 1902. 123-134 p.

6. Young J. Rational treatment of infraclusion. Intern J Orthod. 1923;9(1):1.

7. Gilpatric WH. Arch Predetermination-Is it Practical? J Am Dent Assoc [Internet]. 1923;10(7):553-72. Available from: http://linkinghub.elsevier.com/retrieve/pii/S1048636423070020

8. Neff CW. Tailored Occlusion with the anterior coefficient. merican J Orthod. 1949;35(4):309- 
13.

9. Neff CW. The size relationship between the maxillary and mandibular anterior segments of the dental arch. Angle Orthod. 1957;27(3):138-47.

10. Kesling $\mathrm{H}$. The philosophy of the tooth positioning appliance. Am J Orthod Oral Surg. 1945;31(6):297-304.

11. Bolton W. The clinical application of a tooth-size analysis. Am J Orthod. 1962;48(7):504-29.

12. Lavelle C. Maxillary and mandibular tooth size in different racial groups and in different occlusal categories. Am J Orthod. 1972;61(1):29-37.

13. Hattab FN, Al-Khateeb S, Sultan I. Mesiodistal crown diameters of permanent teeth in Jordanians. Arch Oral Biol. 1996;41(7):641-5.

14. Santoro M, Ayoub ME, Pardi VA, Cangialosi TJ. Mesiodistal Crown Dimensions and Tooth Size Discrepancy of the Permanent Dentition of Dominican Americans. Angle Orthod. 2000;70(4):303-7.

15. Bishara SE, Jakobsen ? JR, Abdallah EM, Fernandez Garcia A. Comparisons of mesiodistal and buccolingnal crown dimensions of the permanent teeth in three populations from Egypt, Mexico, and the United States. Am J Orthod Dentofac Orthop. 1989;96(5):416-22.

16. Smith S, Buschang P, Watanabe E. Interarch tooth size relationships of 3 populations: "does Bolton's analysis apply?" Am J Orthod Dentofacial Orthop [Internet]. 2000;117(2):169-74. Available from: http://www.ncbi.nlm.nih.gov/pubmed/10672217

17. Dempsey P, Townsend GC. Genetic and environmental contributions to variation in human tooth size. Heredity (Edinb). 2001;86(September 2000):685-93.

18. Hughes T, Dempsey P, Richards L, Townsend G. Genetic analysis of deciduous tooth size in Australian twins. Arch Oral Biol. 2000;45(11):997-1004.

19. Bernabé E, Major PW, Flores-Mir C. Tooth-width ratio discrepancies in a sample of Peruvian adolescents. Am J Orthod Dentofac Orthop. 2004;125(3):361-5.

20. Al-Khateeb S, Abu Alhaija E. Tooth size discrepancies and arch parameters among different malocclusions in a Jordanian sample. Angle Orthod. 2006;76(3):459-65.

21. Al-Omari I, Al-Bitar Z, Hamdan A. Tooth size discrepancies among Jordanian schoolchildren. Eur J Orthod. 2008;30(5):527-31.

22. O'Mahony G, Millett DT, Barry MK, Mclntyre GT, Cronin MS. Tooth size discrepancies in Irish orthodontic patients among different malocclusion groups. Angle Orthod. 2011;81(1):130-3.

23. Kumar $P$, Singh V, Kumar $P$, Sharma $P$, Sharma R. Effects of premolar extractions on Bolton overall ratios and tooth-size discrepancies in a Japanese orthodontic population. J Orthod Sci. 2013;2(1):23-7.

24. Endo T, Abe R, Kuroki H, Oka K, Shimooka S. Tooth size discrepancies among different malocclusions in a Japanese orthodontic population. Angle Orthod. 2008;78(6):994-9.

25. Manopatanakul S, Watanawirun N. Comprehensive intermaxillary tooth width proportion of Bangkok residents. Braz Oral Res [Internet]. 2011;25(2):122-7. Available from: http://www.scielo.br/scielo.php?script=sci_arttext\&pid=S1806$83242011000200005 \& \operatorname{lng}=$ en\&tlng=en

26. Ricci ID, Scanavini MA, Kaieda AK, Rosário HD, Paranhos LR. Bolton ratio in subjects with normal occlusion and malocclusion. Brazilian J Oral Sci. 2013;12(4):357-61.

27. Higgins JPT, Thompson SG, Deeks JJ, Altman DG. Measuring inconsistency in meta-analyses. 
BMJ Br Med J. 2003;327(7414):557-60.

28. Liberati A, Altman DG, Tetzlaff J, Mulrow C, Gøtzsche PC, loannidis JPA, et al. The PRISMA statement for reporting systematic reviews and meta-analyses of studies that evaluate health care interventions: Explanation and elaboration. PLoS Med. 2009;6(7).

29. Beller EM, Glasziou PP, Altman DG, Hopewell S, Bastian H, Chalmers I, et al. PRISMA for Abstracts: Reporting Systematic Reviews in Journal and Conference Abstracts. PLoS Med. 2013;10(4).

30. Bugaighis I, Karanth D, Borzabadi-Farahani A. Tooth size discrepancy in a Libyan population, a cross-sectional study in schoolchildren. J Clin Exp Dent. 2015;7(1):e100-5.

31. Alkofide $\mathrm{E}$, Hashim $\mathrm{H}$. Intermaxillary tooth size discrepancies among different malocclusion classes: a comparative study. J Clin Pediatr Dent [Internet]. 2002;26(4):383-7. Available from: http://jocpd.org/doi/10.17796/jcpd.26.4.j46352g860700614\%5Cnhttp://www.ncbi.nlm.nih.g ov/pubmed/12175133

32. Mahmoud N, Eltahir H, Mageet A. Tooth size discrepancy among different malocclusion groups in Sudanese sample. J Orthod Endod. 2017;3(3:10):1-6.

33. Cançado RH, Gonçalves Júnior W, Valarelli FP, Freitas KMS, Crêspo JAL. Association between Bolton discrepancy and Angle malocclusions. Brazilian Oral Res Res. 2015;29(1):1-6.

34. Al-Duliamy MJ, Othman SS, Hussien FA. Comparison of Bolton's Ratios in a Sample of Iraqi and Egyptian Populations. J baghdad Coll Dent [Internet]. 2016;28(4):172-5. Available from: https://www.iasj.net/iasj?func=article \&ald=118507

35. Shastri D, Singh A, Tandon P. Bolton ratio in a North Indian population with different malocclusions. J Orthod Sci [Internet]. 2015;4(3):83-5. Available from: http://www.jorthodsci.org/text.asp?2015/4/3/83/160243

36. Zerouaoui MF, Bahije L, Zaoui F, Regragui S. Study of variations of the Bolton index in the Moroccan population depending on angle malocclusion class. Int Orthod [Internet]. 2014;12(2):213-21. Available from: http://dx.doi.org/10.1016/j.ortho.2014.03.003

37. Asma A. Comparison of anterior tooth size discrepancies among different malocclusion groups. Malaysian J Med Heal Sci. 2013;9(1):73-9.

38. Kansal A, Kittur N, Keluskar KM. Analysis of Bolton's ratio among different malocclusion groups: A hospital based study. Indian J Dent [Internet]. 2012;3(3):139-44. Available from: http://linkinghub.elsevier.com/retrieve/pii/S0975962X12000299

39. Crosby DR, Alexander CG. The occurrence of tooth size discrepancies among different malocclusion groups. Am J Orthod Dentofac Orthop. 1989;95(6):457-61.

40. Gaddam R, Arya S, Shetty K. Incidence of tooth size discrepancy in different malocclusion and relation to extraction. J Int Oral Heal J Int Oral Heal. 2015;77(Suppl1):48-53.

41. Begum M, Goje SK, Karra A, Mohan S. Tooth size and arch parameter discrepancies among different malocclusions in young permanent dentition of 13-15-year-old school children of Nalgonda District-South Indian population. J Orthod Res. 2014;2(1):4-10.

42. Chugh V, Tandon P, Sharma V, Nagar A, Maurya R, Chugh A. An evaluation of tooth size discrepancies among different malocclusion groups in North Indians. J Orthod Res. 2015;3(2):119-23.

43. McSwiney TP, Millett DT, Mclntyre GT, Barry MK, Cronin MS. Tooth size discrepancies in Class II division 1 and Class III malocclusion requiring surgical-orthodontic or orthodontic treatment. J Orthod [Internet]. 2014;41(2):118-23. Available from: 
http://www.ncbi.nlm.nih.gov/pubmed/24526719

44. Ismail MA, Abuaffan AH. Tooth size discrepancy among different malocclusion groups in Sudanese sample. Orthod Waves [Internet]. 2015;74(2):37-41. Available from: http://dx.doi.org/10.1016/j.odw.2015.01.001

45. Hashim AA, Eldin A-H, Hashim H. Bolton tooth size ratio among Sudanese Population sample: A preliminary study. J Orthod Sci [Internet]. 2015;4(3):77-82. Available from: http://www.jorthodsci.org/text.asp?2015/4/3/77/160240

46. Elsheikhi F, Bugaighis I, Hamed T. Tooth size discrepancy in a different malocclusion groups in Libya: a pilot study. Libyan Int Med Univ J [Internet]. 2017;2(2):92-100. Available from: http://journal.limu.edu.ly/index.php/LIJCR/article/view/102

47. Patel $\mathrm{Y}$, Nair $\mathrm{V}$, Jamenis $\mathrm{S}$. Bolton analysis of the maratha population in Pune. J Dent Allied Sci [Internet]. 2017;6(1):8-11. Available from: http://www.jdas.in/text.asp?2017/6/1/8/205439

48. Shahid F, Alam MK, Khamis MF. Intermaxillary tooth size discrepancy in a Pakistani population: A stereomicroscope versus digital caliper. Eur J Dent. 2016;10(2):176-82.

49. Lee SJ, Ahn SJ, Lim WH, Lee S, Lim J, Park HJ. Variation of the intermaxillary tooth-size relationship in normal occlusion. Eur J Orthod. 2011;33(1):9-14.

50. Endo T, Ishida K, Shundo I, Sakaeda K, Shimooka S. Effects of premolar extractions on Bolton overall ratios and tooth-size discrepancies in a Japanese orthodontic population. Am J Orthod Dentofac Orthop. 2010;137(4):508-14.

51. Endo T, Uchikura K, Ishida K, Shundo I, Sakaeda K, Shimooka S. Thresholds for clinically significant tooth-Size discrepancy. Angle Orthod. 2009;79(4):740-6.

52. Nie $Q$, Lin J. Comparison of anterior tooth size discrepancies among different malocclusion groups. Am J Orthod Dentofac Orthop. 1999;116(5):539-44.

53. Saritha T, Sunitha C, Kumar PK, Naveen R. Applicability of Bolton's Analysis to a South Telangana Population. Indian J Dent Sci. 2017;9(4):225-32.

54. Maurya R, Gupta A, Garg J, Mishra HA. Seventh key of occlusion: Diagnostic significance in different angle's class I, II and III malocclusions. J Orthod Res. 2015;3(3):188-91.

55. Jindal R, Bunger E. Bolton 's intermaxillary tooth size ratios among school going children in Punjab population. Indian J Oral Sci. 2013;4(3):110-3.

56. Hyder M, Mamun M, Hossain M. Tooth Size Discrepancies among Different Malocclusions in a Bangladeshi Orthodontic population. Bangladesh J Orthod Dentofac Orthop. 2012;2(2):8-17.

57. Al Sulaimani FFH Al, Afify AR. Bolton Analysis in Different Classes of Malocclusion in a Saudi Arabian Sample. Egyption Dent J. 2006;52(119):1119-25.

58. Ta TA, Ling JYK, Hägg U. Tooth-size discrepancies among different occlusion groups of southern Chinese children. Am J Orthod Dentofac Orthop. 2001;120(5):556-8.

59. Sakoda KL, Pinzan A, Janson G, Cury SEN. Applicability of Bolton's tooth size ratios in Mediterranean, Japanese and Japanese-Brazilian populations. Brazilian J Oral Sci. 2016;15(4):269-72.

60. Jóias RP, Velasco LG, Scanavini MA, Miranda ALR, Siqueira DF. Evaluation of the Bolton ratios on 3D dental casts of Brazilians with natural, normal occlusions. World J Orthod [Internet]. 2010;11(1):67-70. Available from:

http://ovidsp.ovid.com/ovidweb.cgi?T=JS\&PAGE=reference\&D=med5\&NEWS=N\&AN=202091 80 
61. Jóias RP, Scanavini MA. Factors related to Bolton's anterior ratio in Brazilians with natural normal occlusion. Brazilian J Oral Sci. 2011;10(1):69-73.

62. Fernandes TMF, Janson G, Pinzan A, Sathler R, de Freitas LMA, de Freitas MR. Applicability of Bolton tooth-size ratios in racial groups. World J Orthod. 2010;11(4):e57-62.

63. Freire SM, Nishio C, Mendes A de M, Quintó CCA, Almeida MA. Relationship between dental size and normal occlusion in Brazilian patients. Braz Dent J. 2007;18(3):253-7.

64. Carreiro LS, Santos-Pinto A, Raveli DB, Martins LP. A discrepância de tamanho dentário, de Bolton, na oclusão normal e nos diferentes tipos de más oclusões, bem como sua relação com a forma de arco e o posicionamento dentário. R Dent Press Ortodon Ortop Facial. 2005;10(3):97-117.

65. Vela E, Taylor RW, Campbell PM, Buschang PH. Differences in craniofacial and dental characteristics of adolescent Mexican Americans and European Americans. Am J Orthod Dentofac Orthop [Internet]. 2011;140(6):839-47. Available from: http://dx.doi.org/10.1016/j.ajodo.2011.04.026

66. Araujo E, Souki M. Bolton Anterior Tooth Size Discrepancies Among Different Malocclusion Groups. Angle Orthod. 2003;73(3):307-13.

67. Machado V, Botelho J, Pereira D, Vasques M, Fernandes-retto P, Proença L. Bolton ratios in Portuguese subjects among different malocclusion groups. J Clin Exp Dent. 2018;

68. Lombardo L, Marcon M, Arveda N, La Falce G, Tonello E, Siciliani G. Preliminary biometric analysis of mesiodistal tooth dimensions in subjects with normal occlusion. Am J Orthod Dentofac Orthop [Internet]. 2016;150(1):105-15. Available from: http://dx.doi.org/10.1016/j.ajodo.2015.12.021

69. Celikoglu M, Nur M, Kilkis D, Sezgin OS, Bayram M. Mesiodistal tooth dimensions and anterior and overall Bolton ratios evaluated by cone beam computed tomography. Aust Orthod J. 2013;29(2):11-6.

70. Oktay H, Ulukaya E. Intermaxillary tooth size discrepancies among different malocclusion groups. Eur J Orthod. 2010;32(3):307-12.

71. Ciğer S, Aksu M, SaĞlam B. Interarch Tooth-Size Relationships of Normal Occlusion and Class II Division 1 Malocclusion Patients in a Turkish Population Türk Populasyonunda Sınıf II Divizyon 1 Maloklüzyon ve Normal Okluzyonlu Bireylerde İnterark Diş Boyutu iliş̧ileri. Hacettepe Dişhekimliği Fakültesi Derg. 2006;30(4):25-32.

72. Uysal T, Sari Z, Basciftci FA, Memili B. Intermaxillary tooth size discrepancy and malocclusion: Is there a relation? Angle Orthod. 2005;75(2):208-13.

73. Strujic M, Anić-Milošević S, Meštrović S, Šlaj M. Tooth size discrepancy in orthodontic patients among different malocclusion groups. Eur J Orthod. 2009;31(6):584-9.

74. Laino A, Quaremba G, Paduano S, Stanzione S. Prevalence of tooth size dicrepancy among different malocclusion groups. Prog Orthod. 2003;4(1):37-44.

75. Celikoglu M, Nur M, Kilkis D, Sezgin OS, Bayram M. Mesiodistal tooth dimensions and anterior and overall Bolton ratios evaluated by cone beam computed tomography. Aust Orthod J. 2013;29(2):11-6.

76. Endo T, Shundo I, Abe R, Ishida K, Yoshino S, Shimooka S. Applicability of Bolton's tooth size ratios to a Japanese orthodontic population. Odontology. 2007;95(1):57-60.

77. Devi LB, Singh OB, Laiphangbam J. Evaluation of anterior and overall tooth size ratios by Bolton's analysis in North Eastern Indian population. J Pierre Fauchard Acad (India Sect 
[Internet]. 2017;31(2-4):47-50. Available from:

http://linkinghub.elsevier.com/retrieve/pii/S0970219917300115

78. Kumar P, Chitra P. Determination of Bolton Norms for Indian Population Sample. Orthod J Nepal. 2017;7(1):33-6.

79. Subbarao V V., Regalla RR, Santi V, Anita G, Kattimani VS. Interarch tooth size relationship of indian population: Does bolton's analysis apply? J Contemp Dent Pract. 2014;15(1):103-7.

80. Anil S, Monika M. Bolton Analysis of Himachali Ethnic Population. Indian J Dent Sci. 2010;2(4):12-4.

81. Mirzakouchaki B, Shahrbaf S, Talebiyan R. Determining tooth size ratio in an Iranian-Azari population. J Contemp Dent Pract. 2007;8(7):1-5.

82. Poosti M, Jalali T. Tooth size and arch dimension in uncrowded versus crowded class I malocclusions. J Contemp Dent Pract. 2007;8(3):1-8.

83. Kachoei M, Ahangar-Atashi MH, Pourkhamneh S. Bolton's intermaxillary tooth size ratios among Iranian schoolchildren. Med Oral Patol Oral Cir Bucal. 2011;16(4):568-72.

84. Kumar MS, Mohan AM, Kommi PB, Venkatesan R, Suresh V, Kumar KA. Evaluation of Bolton ' $s$ Discrepancy in Un-treated Angles Class I Patients in Pondicherry population : A CrossSectional Study. J Int Oral Heal. 2015;7(12):86-9.

85. Luu NS, Nikolcheva LG, Retrouvey J-M, Flores-Mir C, El-Bialy T, Carey JP, et al. Linear measurements using virtual study models. A systematic review. Angle Orthod [Internet]. 2012;82(6):1098-106. Available from: http://www.angle.org/doi/10.2319/110311-681.1

86. Aragón MLC, Pontes LF, Bichara LM, Flores-Mir C, Normando D. Validity and reliability of intraoral scanners compared to conventional gypsum models measurements: A systematic review. Eur J Orthod. 2016;38(4):1-6.

87. Fleming PS, Marinho V, Johal A. Orthodontic measurements on digital study models compared with plaster models: A systematic review. Orthod Craniofacial Res. 2011;14(1):116.

88. De Luca Canto G, Pachêco-Pereira C, Lagravere MO, Flores-Mir C, Major PW. Intra-arch dimensional measurement validity of laser-scanned digital dental models compared with the original plaster models: A systematic review. Orthod Craniofacial Res. 2015;18(2):65-76.

89. Ferreira JB, Christovam IO, Alencar DS, DaMotta AFJ, Mattos CT, Cury-Saramago A. Accuracy and reproducibility of dental measurements on tomographic digital models: A systematic review and meta-analysis. Dentomaxillofacial Radiol. 2017;46(7). 